\title{
Dissecting the role of initial collision geometry for jet quenching observables in relativistic heavy ion collisions
}

\author{
Jiangyong Jia(贾江涌) $)^{1,2}$, 因 and Rui Wei(魏睿 $)^{1}$ \\ ${ }^{1}$ Department of Chemistry, Stony Brook University, Stony Brook, NY 11794, USA \\ ${ }^{2}$ Physics Department, Brookhaven National Laboratory, Upton, NY 11796, USA
}

(Dated: November 19, 2018)

\begin{abstract}
The observation of large azimuthal anisotropy or $v_{2}$ for hadrons above $p_{T}>5 \mathrm{GeV} / c$ in $\mathrm{Au}+\mathrm{Au}$ collisions at $\sqrt{s_{\mathrm{nn}}}=200 \mathrm{GeV}$ has been a longstanding challenge for jet quenching models based on perturbative QCD (pQCD). Using a simple jet absorption model, we seek to clarify the situation by exploring in detail how the calculated $v_{2}$ varies with choices of the collision geometry as well as choices of the path length dependence and thermalization time $\tau_{0}$ in the energy loss formula. Besides the change of eccentricity due to distortion from gluon saturation or event-by-event fluctuation, we find that the $v_{2}$ is also sensitive to the centrality dependence of multiplicity and the relative size between the matter profile and the jet profile. We find that the $v_{2}$ calculated for the naive quadratic path length dependence of energy loss, even including eccentricity fluctuation and the gluon saturation, is not enough to describe the experimental data at high $p_{T}(\sim 6 \mathrm{GeV} / c)$ in $\mathrm{Au}+\mathrm{Au}$ collisions. However, it can match the full centrality dependence of $v_{2}$ data if higher power path length dependence of energy loss is allowed. We also find that the calculated $v_{2}$ is sensitive to the assumption of the early time dynamics but generally increases with $\tau_{0}$, opposite to what one expects for elliptic flow. This study attests to the importance of confining the initial geometry, possibly by combining jet quenching $v_{2}$ with elliptic flow and other jet quenching observables, for proper interpretation of the experimental data.
\end{abstract}

PACS numbers: 25.75.-q

\section{INTRODUCTION}

After the discovery of strongly interacting Quark Gluon Plasma (sQGP) at the Relativistic Heavy Ion Collider (RHIC) in 2005 [1], the focus of the heavy ion community shifted toward a detailed characterization of the properties of the sQGP. One of the primary tools is jet quenching or the suppression of high transverse momentum $\left(p_{T}\right)$ hadron yields as a result of in-medium radiative energy loss of high $p_{T}$ jets [2-4]. Due to the large momentum scale of the jets and asymptotic freedom of Quantum Chromodynamics (QCD), jet quenching is usually thought to be described by the pertubative QCD (pQCD) framework, which assumes that jets couple weakly with the medium, even though the medium itself is strongly coupled. Jet quenching models based on pQCD have been developed to describe measurements on single hadron yield [5, 6], di-hadron correlation [7, 8], and $\gamma$-hadron correlation 9 12]. Initial estimates of the properties of sQGP, such as the momentum broadening per mean free path, $\hat{q}=\left\langle k_{T}^{2}\right\rangle / \lambda$, and energy loss per unit length, $d E / d l$, have been obtained [13].

Despite its early successes, the pQCD description of jet quenching faces several challenges (see Ref [14]). One observable that has thus far defied the pQCD description is high $p_{T} v_{2}$ or azimuthal anisotropy of particles emitted relative to the reaction plane $(\mathrm{RP})$ in $\mathrm{Au}+\mathrm{Au}$ collisions, $d N / d\left(\phi-\Psi_{\mathrm{RP}}\right) \propto\left(1+2 v_{2} \cos 2\left(\phi-\Psi_{\mathrm{RP}}\right)\right)$.

\footnotetext{
*Correspond to jjia@bnl.gov
}

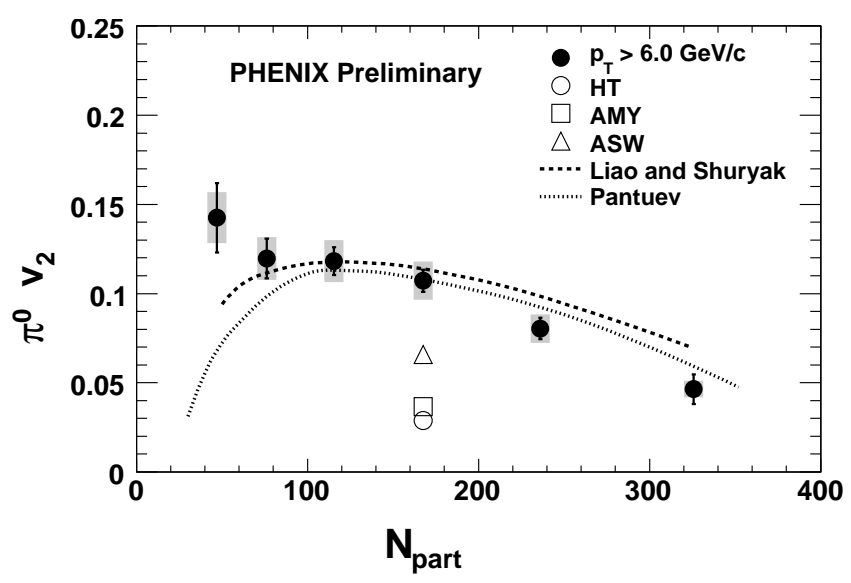

FIG. 1: Figure adapted from Ref. [18]. Data points: PHENIX $\pi^{0} v_{2}$ at $p_{T}>6 \mathrm{GeV} / c$; open symbols: three pQCD model calculations taken from Ref. [13]; lines: geometric model calculations with different assumptions on path-length dependence [25, 59].

Such azimuthal anisotropy ensues because the jet yield is more suppressed along the long axis of the fireball (out-of-plane) than the short axis (in-plane). Thus, the $v_{2}$ value is sensitive to the path length $(l)$ dependence of energy loss, which scales, in the pQCD framework, as $\Delta E \propto l$ and $\Delta E \propto l^{2}$ for elastic and LandauPomeanchuk-Migdal(LPM) radiative energy loss [15], respectively. Currently, most pQCD models undershoot the $v_{2}$ value by as much as factor of 2 in the experimentally accessible $p_{T}$ range $\left(p_{T}<10 \mathrm{GeV} / c\right)$ [16, 17]. We illustrate this situation with Fig. 1 borrowed from 
Ref. [18], which compares three mainstream pQCD model calculations (abbreviated as AMY, ASW, and HT) 13] with recent precision PHENIX data at $p_{T} \sim 6 \mathrm{GeV} / c$.

This, together with its failure in describing heavy flavor suppression [19], call into question the perturbative assumption used in the pQCD framework. It may happen that the coupling between the jet and the medium for typical RHIC jet energy of $p_{T} \lesssim 10-20 \mathrm{GeV} / c$ is still strong enough [20], such that path length dependence and the color charge dependence are modified from pQCD expectation. In fact, calculation based on anti-de Sitter/conformal field theory (AdS/CFT) technique for strongly coupled plasma suggests that $\Delta E \propto l^{3}$ [21, 22] and $\hat{q} \propto \sqrt{\alpha_{\mathrm{SYM}} N_{c}}$ 23], instead of $\Delta E \propto l^{2}$ and $\hat{q} \propto$ $\alpha_{s} N_{c}^{2}$ for pQCD. This higher order path length dependence could explain the large anisotropy [24]. Liao and Shuryak 25] argue that most energy loss in sQGP is concentrated around $T_{c}$; such a non-monotonic dependence of energy loss with energy density apparently achieves better description of the data, as shown by Fig. 1.

It is tempting to conclude from this discussion that the data favor a $l$ dependence stronger than the naive $\Delta E \propto l^{2}$ implied by the pQCD radiative energy loss. However, as was pointed out in Ref. [17], the magnitude of the anisotropy is also very sensitive to the choice of initial collision geometry, which is poorly constrained. The collision geometry used by most jet quenching calculations is obtained from the so-called Optical Glauber model [26], which assumes a smooth Woods-Saxon nuclear geometry for $\mathrm{Au}$ ions. It ignores two important modifications: an event-by-event distortion of the shape of the overlap from random fluctuation of positions of participating nucleons [28]; and a possible overall distortion of the shape of the overlap due to, e.g. gluon saturation effect (so called CGC geometry [27]). Both effects are shown to lead to $15 \%-30 \%$ corrections in the hydrodynamic calculation of elliptic flow at low $p_{T}$ [30]; they were also shown in Refs. 27, 31] to play an important role for jet quenching calculation of azimuthal anisotropy at high $p_{T}$.

Furthermore, the way that collision geometry influence the jet quenching $v_{2}$ is quite different from that for hydrodynamic description of low $p_{T} v_{2}$. Hydrodynamic flow is a self generating process driven by the shape or eccentricity $\left(\epsilon=\frac{\left\langle y^{2}\right\rangle-\left\langle x^{2}\right\rangle}{\left\langle y^{2}\right\rangle+\left\langle x^{2}\right\rangle}\right)$ of a single matter profile, i.e. $v_{2} \propto \epsilon$; whereas the $v_{2}$ from jet quenching requires both the profile for the bulk matter AND the jet production points. The two profiles may not necessarily have the same spatial distribution because various nuclear effects at initial state may induce sizable momentum (e.g., Bjorken momentum fraction $2 p_{T} / \sqrt{s}$ ) and position dependent modification, analogous to the generalized parton distribution for proton. Hence high $p_{T} v_{2}$ depends not only on the eccentricity of the fireball, but also on the matching (relative size and shape) between the jet and the matter profiles. Understanding the role of geometry and scaling behavior of the data such as those in Ref. [32, 33] is important for proper interpretation of the experimental data.

In this article, we investigate the sensitivity of the jet quenching $v_{2}$ on the choices and uncertainties of the collision geometry for the bulk matter. We check explicitly the scaling and violation thereof with the bulk eccentricity. We explore, in the context of these uncertainties, whether the data allow for high order $l$ dependence of energy loss. The prospects of constraining the initial collision geometry using $v_{2}$ and other jet quenching observables, such as single inclusive suppression $R_{\mathrm{AA}}$, inclusive away-side suppression $I_{\mathrm{AA}}$ and associated anisotropy $v_{2}^{I_{\mathrm{AA}}}$, are discussed.

\section{MODEL IMPLEMENTATION}

We generate the Glauber geometry using an improved version of the publicly available PHOBOS code [34]. Each $\mathrm{Au}$ ion is populated randomly with nucleons with a hard-core of $0.3 \mathrm{fm}$ in radii, according to the WoodsSaxon distribution with a radius of $6.38 \mathrm{fm}$ and diffuseness of $0.535 \mathrm{fm}$. A nucleon-nucleon collision is considered to happen when their distance in the $x y$-plane fall within $\sqrt{\sigma_{\mathrm{nn}}^{\text {inel }} / \pi}=1.16 \mathrm{fm}$ (hard-sphere assumption), corresponding a $n-n$ cross-section of $\sigma_{\mathrm{nn}}^{\text {inel }}=42 \mathrm{mb}$. Subsequently, the number density of nucleons participating in the collision $\left(\rho_{\text {part }}(x, y, b)\right)$ and the number density of binary collisions $\left(\rho_{\text {coll }}(x, y, b)\right)$ can be determined in the $x y$-plane as function of impact parameter $b$. Here the $x$ direction is always chosen to be along the line connecting the centers of the two ions. Denoting $T_{A}$ as the thickness function for $\mathrm{Au}$ ion, they can be approximated with the following expression when nucleon size is ignored.

$$
\begin{aligned}
\rho_{\text {part }}(x, y, b) & \approx T_{A}\left(x+\frac{b}{2}, y\right)\left[1-P\left(x+\frac{b}{2}, y\right)\right] \\
& +T_{A}\left(x-\frac{b}{2}, y\right)\left[1-P\left(x+\frac{b}{2}, y\right)\right], \\
\rho_{\text {coll }}(x, y, b) & \approx \sigma_{\text {nn }}^{\text {inel }} T_{A}\left(x+\frac{b}{2}, y\right) T_{A}\left(x-\frac{b}{2}, y\right),(1)
\end{aligned}
$$

where $P(x, y)=\left(1-\frac{\sigma_{\mathrm{nn}}^{\mathrm{inel}} T_{A}(x, y)}{A}\right)^{A}$ and $A=197$ is the number of nucleons in $\mathrm{Au}$ ion.

We generate the CGC geometry using the MC-KLN model by Drescher \& Nara [27, 29], which is based on the well known KLN (Kharzeev-Levin-Nardi) $k_{T}$ factorization approach [35]. In a nutshell, the MC-KLN model calculates the CGC geometry event by event by modifying the output from a Monte-Carlo Glauber model. Specifically, the transverse gluon density profile, $d n / d y(x, y, b)$, is calculated through the $k_{T}$ factorization formula, with the saturation scale $Q_{s}^{2}$ of each Au ion set to be proportional to its thickness function $T_{A}$ or $T_{B}$. To ensure internal consistency, the MC-KLN code is adapted to the same Glauber algorithms as the PHOBOS code (same hardcore nucleons and identical Woods-Saxon parameters). The obtained gluon density scales approximately [27] as 
$\min \left\{T_{A}, T_{B}\right\}$ in the $x$ direction and $1 / 2\left(T_{A}+T_{B}\right)$ in the $y$ direction, which leads to a $20 \%-30 \%$ increase of the eccentricity relative to the Glauber geometry (see left panels of Fig. (5). When implemented in hydrodynamic model calculations [30, 36], a similar amount of increase is seen for the predicted elliptic flow signal.

We account for initial geometry fluctuation in Glauber geometry by re-centering and rotating all participants, such that the "participant plane" (PP), defined as the minor axis direction of all participants (see Ref. [28]), aligns with the lab frame. We then sum all events together to give the overall participant density profile. The same amount of shift and rotation is then applied for all binary collisions to get the overall density profile for jet production points. We repeat the same procedure to the gluon density profile for CGC geometry. Important variables include the orientation of the participant plane $\left(\Psi_{\text {part }}\right)$ for either participants or gluon density ${ }^{1}$, eccentricity with respect to the reaction plane $\left(\epsilon_{\mathrm{RP}}\right)$, eccentricity with respect to the participant plane $\left(\epsilon_{\mathrm{part}}\right)$, and average root mean square (RMS) size of the ellipsoid $\left(\sigma_{r}\right)$. They are calculated for each event as

$$
\begin{aligned}
\tan \left(2 \Psi_{\mathrm{part}}\right) & =\frac{\sigma_{y}^{2}-\sigma_{x}^{2}}{2 \sigma_{x y}^{2}}, \\
\epsilon_{\mathrm{RP}} & =\frac{\sigma_{y}^{2}-\sigma_{x}^{2}}{\sigma_{y}^{2}+\sigma_{x}^{2}}, \\
\epsilon_{\mathrm{part}} & =\frac{\sqrt{\left(\sigma_{y}^{2}-\sigma_{x}^{2}\right)^{2}+4 \sigma_{x y}^{2}}}{\sigma_{y}^{2}+\sigma_{x}^{2}}=\frac{\sigma_{y}^{\prime 2}-\sigma_{x}^{\prime 2}}{\sigma_{y}^{\prime 2}+\sigma_{x}^{\prime 2}}, \\
\sigma_{r}^{2} & =\sigma_{y}^{2}+\sigma_{x}^{2}=\sigma_{y}^{\prime 2}+\sigma_{x}^{\prime 2},
\end{aligned}
$$

where $\sigma_{x}^{2}, \quad \sigma_{y}^{2}$ and $\sigma_{x y}$ are the event-by-event (co)variances of participant density profile for Glauber geometry or gluon density profile for CGC geometry, respectively, and ${\sigma_{x}^{\prime 2}}^{2}$ and $\sigma_{y}^{\prime^{2}}$ are variances defined in the rotated frame. We emphasize that the participant plane angle, $\Psi_{\text {part }}$, should be the natural frame for both hydrodynamic flow and jet quenching. However, it is tilted by a different amount in the case of the CGC geometry from the Glauber geometry.

In this work, the magnitude of the jet quenching $v_{2}$ depends on the following four control factors:

1. Energy loss formula, including the path length dependence, thermalization time etc.

2. Eccentricity, including event-by-event fluctuation

\footnotetext{
${ }^{1}$ We emphasize that the PP (and $\Psi_{\text {part }}$ ) is calculated in the position space of nucleons or gluons in a simulated collision, whereas experiments measure the so called event plane (EP) using final state particles in momentum space of a real collision. Both PP and EP include fluctuations and approximate the true RP of their respective collisions, but they may not coincide with each other.
}

and the shape of collision geometry (e.g. CGC vs Glauber).

3. Centrality dependence of the total multiplicity. Because the jet quenching strength is fixed in most central collisions, if matter density falls faster toward peripheral collisions, we expect less suppression and smaller $v_{2}$ in peripheral collisions.

4. The size of the matter profile relative to the jet profile. If the transverse size of the matter profile is smaller than that for the jet profile, more surviving jets should originate from the corona region, leading to a smaller $v_{2}$.

Clearly, the collision geometry (items 2-4) plays an essential role for proper understanding of the energy loss mechanism (item 1). In contrast to hydrodynamic description of low $p_{T} v_{2}$, which depends only on the eccentricity of the ellipsoid, jet quenching description of high $p_{T} v_{2}$ is sensitive to two more aspects of the collision geometry (items 3 and 4). The primary goal of this work is to understand how the jet quenching $v_{2}$ depends on the underlying choices of eccentricity, centrality dependence of multiplicity, and matching between the matter and the jet profile.

We base the study on the following four matter profiles (three versions of Glauber geometry and one CGC geometry)

$$
\begin{aligned}
\rho_{0}(x, y, b) & =\rho_{\text {part }}(x, y, b) \\
\rho_{1}(x, y, b) & =\rho_{\text {coll }}(x, y, b), \\
\rho_{2}(x, y, b) & =\frac{1-\delta}{2} \rho_{\text {part }}(x, y, b)+\delta \rho_{\text {coll }}(x, y, b), \\
\rho_{3}(x, y, b) & =\rho_{\mathrm{CGC}}(x, y, b)=d n / d y(x, y, b),
\end{aligned}
$$

with the corresponding integral form

$$
\begin{aligned}
\int d x d y \rho_{0}(x, y, b) & =N_{\text {part }}(b) \\
\int d x d y \rho_{1}(x, y, b) & =N_{\text {coll }}(b) \\
\int d x d y \rho_{2}(x, y, b) & =\frac{1-\delta}{2} N_{\text {part }}(b)+\delta N_{\text {coll }}(b) \\
& =d N / d y(b) \\
\int d x d y \rho_{3}(x, y, b) & =d N / d y(b),
\end{aligned}
$$

where $\rho_{\text {part }}$ and $\rho_{\text {coll }}$ are transverse participant density and collision density from the Glauber model, respectively; $\rho_{2}$ is the two component Glauber model from Ref. [37] with $\delta=0.14$ [30], and $\rho_{3}=\rho_{\mathrm{CGC}}$ is the transverse gluon density from MC-KLN. Both $\rho_{2}$ and $\rho_{3}$ have been adjusted [30] such that their total integrals match the centrality dependence of the charged hadron multiplicity, $d N / d y(b)$, at RHIC [38]. For the first two profiles, 
$\rho_{0}$ and $\rho_{1}$, we can enforce the same centrality dependence as $d N / d y(b)$ by applying a centrality dependent scale factor:

$$
\begin{aligned}
\rho_{0}^{\mathrm{Mul}}(x, y, b) & =\frac{d N / d y(b)}{N_{\text {part }}(b)} \rho_{\text {part }}(x, y, b), \\
\rho_{1}^{\mathrm{Mul}}(x, y, b) & =\frac{d N / d y(b)}{N_{\text {coll }}(b)} \rho_{\text {coll }}(x, y, b), \\
\int d x d y \rho_{0}^{\mathrm{Mul}}(x, y, b) & =d N / d y(b) \\
\int d x d y \quad \rho_{1}^{\mathrm{Mul}}(x, y, b) & =d N / d y(b)
\end{aligned}
$$

These scale factors essentially account for the different centrality dependence trends between $N_{\text {part }}$ and $N_{\text {coll }}$ relative to $d N / d y$. Figure 2 shows the rate of change of $d N / d y$ and $N_{\text {coll }}$ relative to $N_{\text {part }}$ normalized to unity for most central points. Clearly, the $N_{\text {coll }}$ has the fastest change vs. centrality, followed by $d N / d y$, and $N_{\text {part }}$ has the slowest change vs. centrality. Nevertheless, the resulting profiles, $\rho_{0}^{\mathrm{Mul}}$ and $\rho_{1}^{\mathrm{Mul}}$, still maintain their original shape and size. They are used to study the sensitivity of $v_{2}$ to the centrality dependence of the multiplicity.

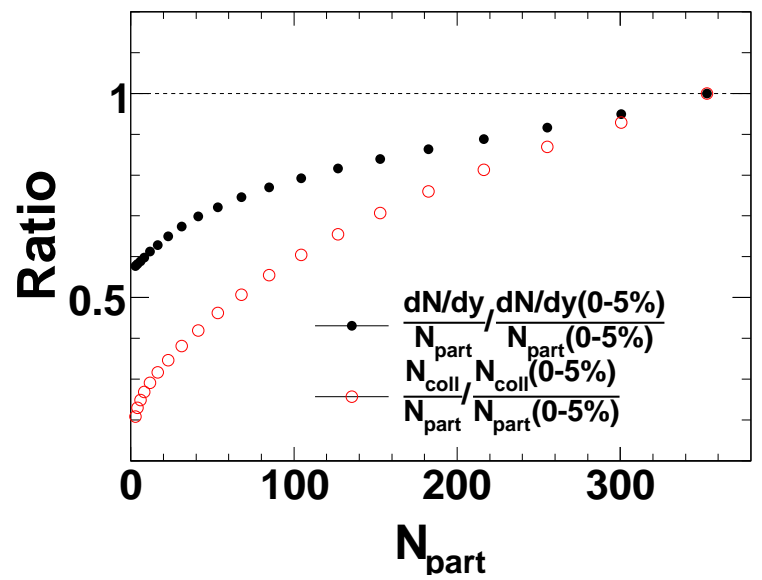

FIG. 2: (Color online) The centrality dependence of $d N / d y / N_{\text {part }}$ (filled) and $N_{\text {coll }} / N_{\text {part }}$ (open) normalized by their values in most central $0 \%-5 \%$ bin. This plot illustrates different rates of change as a function of centrality, between $N_{\text {part }}, N_{\text {coll }}$, and $d N / d y$.

Besides the two profiles obtained by matching to $d N / d y$, we are also interested in several other variants of $\rho_{0}-\rho_{3}$, obtained either by rotation of their respective participant planes ${ }^{2}$,

$$
\begin{aligned}
\rho_{n}^{\operatorname{Rot}}(x, y)= & \rho_{n}\left(x \cos \Psi_{\text {part }}-y \sin \Psi_{\text {part }},\right. \\
& \left.x \sin \Psi_{\text {part }}+y \cos \Psi_{\text {part }}\right),
\end{aligned}
$$

or by readjusting the overall size by a constant scale factor $a$ to match to that of $\rho_{0}$ (see discussions in Secs. III B and IIC)

$$
\rho_{n}^{\text {Resize }}(x, y)=\rho_{n}(a x, a y),
$$

or by a combination of matching multiplicity, rotation or readjusting size. In the case that a profile is obtained via several operations, we use appropriate superscript to

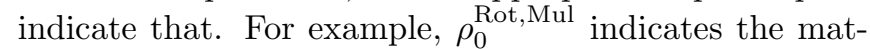
ter profile obtained by rotating the event-by-event participant profile according to the participant plane angle, followed by matching its total integral to $d N / d y$ for each centrality bin. Note the order of these operations has no significance because they factorize.

We implement jet quenching using the simple jet absorption model of Ref. [17]. It provides a transparent way of investigating the sensitivity of jet quenching observables to choices of the collision geometry. In this model, back-to-back jet pairs are generated according to the binary collision density profile in $x y$-plane with uniform orientation. These jets are then propagated through the medium whose density is given by matter profile $\rho(x, y)$, with a survival probability $e^{-\kappa I}$. In the default setup, matter integral $I$ is calculated as

$I=\int_{0}^{\infty} d l \frac{l}{l+l_{0}} \rho\left(\overrightarrow{\mathbf{r}}+\left(l+l_{0}\right) \widehat{\mathbf{v}}\right) \approx \int_{0}^{\infty} d l \rho(\overrightarrow{\mathbf{r}}+l \widehat{\mathbf{v}})(5)$

for a jet generated at $\overrightarrow{\mathbf{r}}=(x, y)$ and propagated along direction $\widehat{\mathbf{v}}$. This corresponds to a quadratic dependence of absorption $(\propto l d l)$ in a longitudinal expanding or $1+1 \mathrm{D}$ medium $\left(\propto 1 /\left(l_{0}+l\right)\right)$ with a thermalization time of $l_{0}=c \tau_{0}$. It is fixed to 0 by default but we explore non-zero value of $l_{0}$ in Sec. IIE, The absorption coefficient $\kappa$ (which controls the jet quenching strength) is chosen to reproduce $R_{\mathrm{AA}}=\left\langle e^{-\kappa I}\right\rangle \sim 0.18$ for $0 \%$ $5 \% \pi^{0}$ data [41]. We explore path length dependence by extending Eq. 5 to four different functional forms,

$$
I_{m}=\int_{0}^{\infty} d l l^{m-1} \rho(\overrightarrow{\mathbf{r}}+l \widehat{\mathbf{v}}), \quad m=1,2,3,4,
$$

where $m=1$ and $m=2$ correspond to $l$ dependence for radiative, and AdS/CFT energy loss in 1+1D medium, respectively.

A typical calculation starts by choosing one of the four

\footnotetext{
2 Note that the participant plane angle $\Psi_{\text {part }}$ is defined separately for $\rho_{0}-\rho_{3}$. For $\rho_{1}$, it is determined by the minor axis of all binary collisions, while that for $\rho_{2}$ is determined by both participants and binary collision with appropriate weights given in Eq. 3
} 
matter profiles (Glauber geometry $\rho_{0}-\rho_{2}$ or CGC geometry $\rho_{3}$ ) and applying appropriate modifications (specifying Rot, Mul, and/or Resize). We specify the jet absorption scheme by varying thermalization time $l_{0}$ or the order of path length dependence $m$. We then fix the $\kappa$ value by matching $R_{\mathrm{AA}} \sim 0.18$ in most central collision (We explore the uncertainties of $\kappa$ arising from experimental uncertainties of $R_{\mathrm{AA}}$, and discuss their implications in Appendix $(\mathrm{B})$. On the other hand, the jet production profile is always given by $\rho_{\text {coll }}$. We stress that $\kappa$ is the only free parameter, and has similar role as the $\hat{q}$, and it is tuned independently for each one of these running modes (there are $\sim 100$ of them, depending on the choice of matter profiles, $m$, and modifications of matter profiles). Once $\kappa$ is known, we can predict the centrality dependence of the single hadron suppression $\left(R_{\mathrm{AA}}\right)$, jet quenching $v_{2}$ which can be expressed as $v_{2}=\left\langle e^{-\kappa I} \cos 2\left(\phi-\Psi_{\text {part }}\right)\right\rangle$; and away-side per-trigger yield suppression $\left(I_{\mathrm{AA}}\right)$.

Finally, we point out that the $v_{2}$-like modulation is found to be the dominating contribution to the azimuthal anisotropy obtained in our calculations. The higher order terms, mostly $v_{4}$, are found to be less than $10 \%$ of $v_{2}$ value for all running modes. Thus we can safely assume that the azimuthal distribution of particle production relative to the PP angle follows a $1+2 v_{2} \cos 2\left(\phi-\Psi_{\text {part }}\right)$ shape (for example see Fig. 19).

\section{RESULTS}

\section{A. Glauber geometry based on participant profile}

As mentioned previously, this work investigates three versions of Glauber geometry, participant profile $\rho_{0}$, collisional profile $\rho_{1}$ and two component profile $\rho_{2}$ and their variants. $\rho_{0}$ is our default Glauber geometry and is the topic of this section; we shall discuss $\rho_{1}$ and $\rho_{2}$ in Section IIIC

Figure 3 shows the $v_{2}$ calculated for Glauber geometry $\rho_{0}^{\mathrm{Mul}}$, that is, participant profile scaled to match the experimental multiplicity. Results are presented in left panel for four different path length dependencies ( $I_{1}-I_{4}$ from bottom to top) with (solid lines) and without (dashed lines) taking into account the fluctuation of PP angle. They are compared with the PHENIX $\pi^{0} v_{2}$ data integrated above $6 \mathrm{GeV} / c$ from Fig 1. The right panel shows the ratios of calculated $v_{2}$ for $I_{1}-I_{4}$ (solid lines) and the ratio of the eccentricity (open circles) between with and without including the fluctuations.

We see that increasing $m$ (the order of $l$ dependence) significantly increases the $v_{2}$ for mid-central collisions, but they all systematically under-predict the data toward central collisions. In fact, the calculated $v_{2}$ for central collision is insensitive to the functional form of path length dependence, due to the almost isotropic shape of the overlap. This situation is dramatically improved when the fluctuation in the PP angle is included. The relative increase in $v_{2}$ is about $15 \%$ for mid-centrality, and
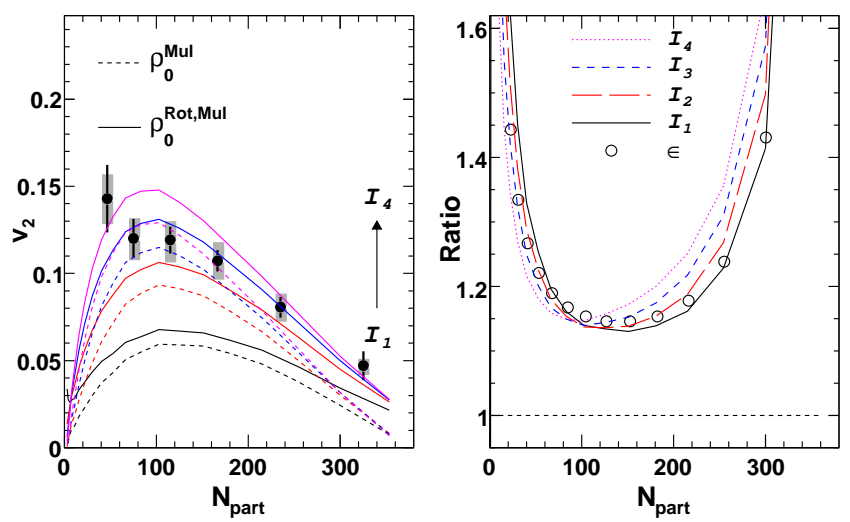

FIG. 3: (Color online) Left: $v_{2}$ calculated with $\rho_{0}^{\mathrm{Mul}}$ (participant density profile scaled to match $\mathrm{dN} / \mathrm{dy}$ ) with (solid lines) and without (dashed lines) rotation to the participant plane compared with data at $6 \mathrm{GeV} / c$ (solid circles) for $I_{1}-I_{4}$ (from bottom up); Right: Corresponding ratios between with and without rotation for $v_{2} \mathrm{~s}$ from $I_{1}-I_{4}$ (lines) and for the eccentricity (open circles).

is significantly larger for central and peripheral collisions. This is consistent with previous studies of low $p_{T} v_{2}$ or elliptic flow, which shows that PP fluctuation needs to be included in $\mathrm{Cu}+\mathrm{Cu}$ and central $\mathrm{Au}+\mathrm{Au}$ collisions in order for hydrodynamic model prediction to work [28, 42]. It is interesting to see that the fractional increase of $v_{2}$ for $I_{1}$ is similar to the fractional increase in eccentricity (i.e., $\epsilon_{\mathrm{part}} / \epsilon_{\mathrm{RP}}$ in the right panel). However, the ratios indicate that the fractional increase for $N_{\text {part }}>50$ is successively larger for larger $m$. This is because larger $m$ places more weight to the large $l$ region; and thus is more sensitive to changes in shape.
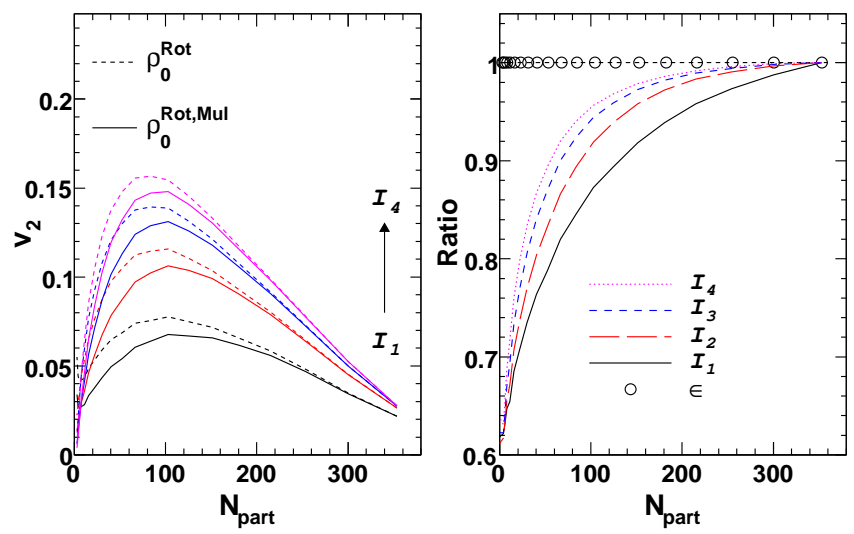

FIG. 4: (Color online) Left: $v_{2}$ calculated with $\rho_{0}^{\text {Rot }}$ (participant density profile rotated to participant plane) with (solid lines) and without (dashed lines) scaling to match $d N / d y$ for $I_{1}-I_{4}$ (from bottom up); Right: Corresponding ratios between with to without scaling to $d N / d y$ for $v_{2}$ s from $I_{1}-I_{4}$ (lines) and for the eccentricity (open circles). Note that the eccentricities are identical for the two cases.

The high $p_{T}$ azimuthal anisotropy, being the result of 
jet quenching, depends not only on the shape, but also on the average density or total multiplicity of the matter profile. To illustrate this point, Fig. 4 shows the $v_{2}$ calculated for matter density $\rho_{0}^{\text {Rot }}$ and $\rho_{0}^{\text {Rot,Mul }}$. They have identical shape and size for each centrality selection, but the integral of $\rho_{0}^{\text {Rot,Mul }}$ drops more rapidly to lower $N_{\text {part }}$. Because jet absorption strength $\kappa$ is tuned to reproduce a common suppression level in central collision, the profile whose average density varies more rapidly with centrality is expected to show less suppression and less $v_{2}$ in peripheral collisions. Indeed, the calculated $v_{2}$ for $\rho_{0}^{\text {Rot,Mul }}$ is smaller than that for $\rho_{0}^{\text {Rot }}$ due to a faster fall off toward peripheral bin.

Figure 4 also shows a weakening of the sensitivity for larger $m$. This is because the weighting from the large $l$ region is reduced in peripheral collisions due to a smaller geometrical size. That reduction is stronger for larger $m$, which leads to smaller sensitivity for large $m$.

One may argue that since $d N / d y(b)$ is constrained by experimental data, there should be no uncertainty associated with the modeling of centrality dependence. However, the matter profiles that were tuned to $\mathrm{Au}+\mathrm{Au} 200$ $\mathrm{GeV}$ data typically shows $\sim 10 \%$ deviation from $\mathrm{Cu}+\mathrm{Cu}$ or $\mathrm{Au}+\mathrm{Au}$ at different collision energies [43, 44]. Furthermore, many current pQCD model calculations use profiles that do not match the $d N / d y$ data. For example, various $1+1 \mathrm{D}$ energy loss models assumes energy loss or $\hat{q}$ to be proportional to either $\rho_{\text {part }}[8,45,46]$ or $\rho_{\text {coll }}$ [47]; Recent more sophisticated calculations [12, 13, 48] based on $3 \mathrm{D}+1$ hydrodynamics model of Nonaka and Bass [49], assume the energy loss or $\hat{q} \propto e^{3 / 4}$ with $e \propto 0.6 \rho_{\text {coll }}+$ $0.4 \rho_{\text {part }}$ [49], which is also different from $d N / d y$. So it seems reasonable to use the difference of the $v_{2}$ in right panel of Fig. 4 as one of the uncertainties in theoretical implementation of initial geometry.

Figure 3 and 4 represent the general style of the presentation of the $v_{2}$ calculation in this article: The left panels always shows the $v_{2}$ values for $I_{1}-I_{4}$ compared between two matter profiles with (solid lines) and without (dashed lines) a particular geometrical effect; the right panels always show the ratios between the two (solid line divided by dashed line). In most cases, the ratio of their eccentricities is shown as open circles on the right panel to compare with $v_{2}$ ratios. Finally, we stress that the jet production profiles are always sampled from $\rho_{\text {coll }}$ throughout this study, so any difference in the calculated $v_{2}$ can be attributed to the differences between the two matter profiles.

\section{B. CGC geometry}

As outlined in the introduction, the MC-KLN model is built on the standard Monte-Carlo Glauber model. So CGC matter profile $\rho_{3}$ contains both the overall modification of shape due to gluon saturation, and the eventby-event fluctuation stemming from participant fluctuation at Glauber level. In addition, we can safely use the same binary collision profile for the jet production, given that the saturation effects are not expected to modify hard processes with momentum transfer well above the saturation scale, $Q^{2} \gg Q_{s}^{2}$.

Another important feature of the CGC geometry via the MC-KLN model is that despite having a larger eccentricity, its overall size is about $4 \%-8 \%$ smaller than the participant profile. One can see it quantitatively in Fig. 5, which compare the eccentricity and overall RMS width $\left(\sigma_{r}\right)$ between $\rho_{3}$ and $\rho_{0}$. This narrowing of CGC geometry was pointed out before by the authors of MCKLN model (see the preprint version of [27]), and can be seen more clearly by plotting the $1 \mathrm{D}$ projections of medium profiles along the $\mathrm{x}$ (in-plane) and $\mathrm{y}$ (out-ofplane) directions (Fig. 6). The projections show that CGC profile is narrower than Glauber profile in both $\mathrm{x}$ and y direction, however since $\sigma_{x}$ is reduced more than $\sigma_{y}$ in MC-KLN vs Glauber, $\epsilon_{\text {part }}$ is larger in MC-KLN.
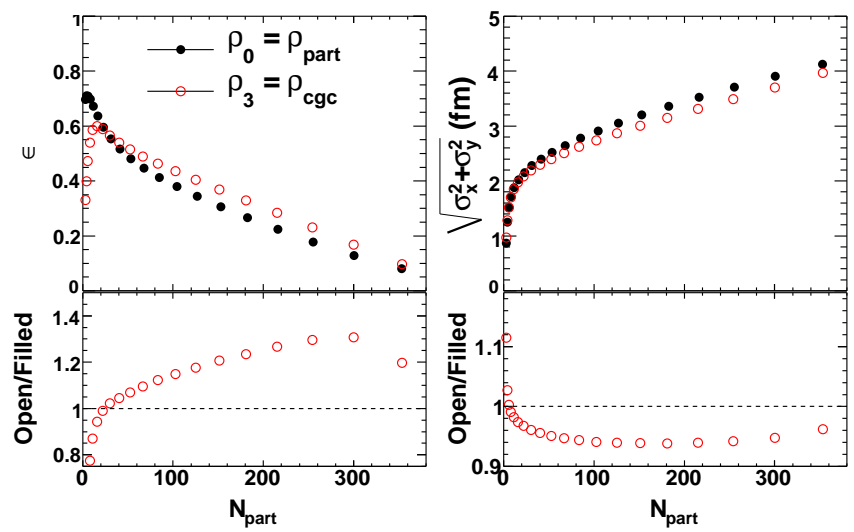

FIG. 5: (Color online) Left: Eccentricities for Glauber geometry calculated from participant density profile $\rho_{0}$ (solid circles) and for CGC geometry $\rho_{3}$ (open circles) in the top panel and the corresponding ratio in the bottom panel. Right: Same as right panels except they are for the RMS size $\sigma_{r}=\sqrt{\sigma_{x}^{2}+\sigma_{y}^{2}}$.

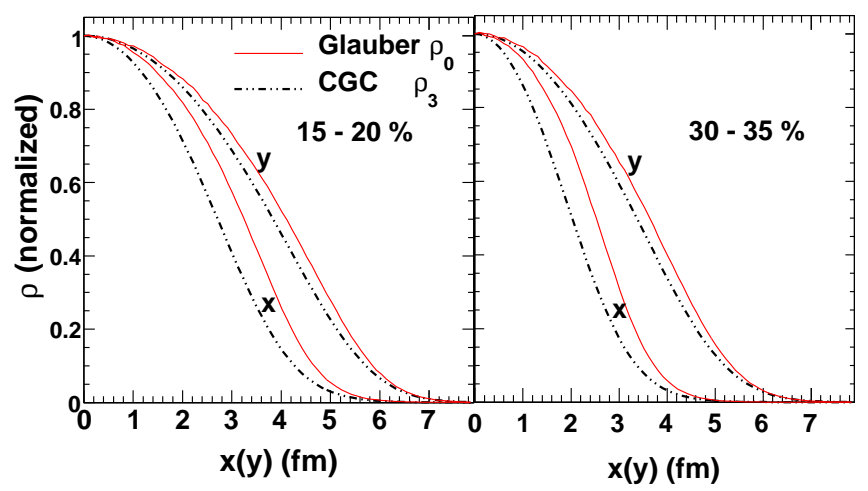

FIG. 6: (Color online) The Glauber geometry $\left(\rho_{0}\right)$ and CGC geometry $\left(\rho_{3}\right)$ projected onto the $x$ and $y$ axes for $15 \%-20 \%$ and $30 \%-35 \%$ centrality bins. They are normalized to 1 at the maximum.

Figure 7 compares the $v_{2}$ calculated for CGC geome- 
try $\left(\rho_{3}\right)$ and Glauber geometry $\left(\rho_{0}\right)$ in their respective rotated frames. The CGC geometry does lead to a larger $v_{2}$; however, the amount of increase is only half of the increase in eccentricity. To check whether the breaking of the eccentricity scaling can be attributed to the $4 \%$ $8 \%$ mismatch between the two profiles, we re-scale the RMS size of the CGC geometry to match that for the Glauber geometry for each centrality while preserving its original shape. Figure 8 shows the same comparison after the scaling is applied and $\kappa$ is re-tuned. The ratios of the calculated $v_{2}$ now match well with the ratio of the eccentricities.
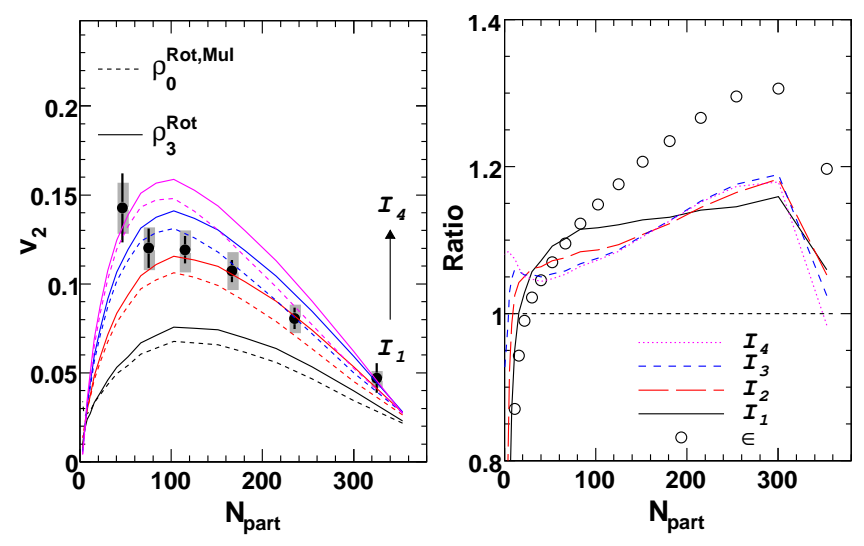

FIG. 7: (Color online) Left: $v_{2}$ calculated for Glauber geometry $\left(\rho_{0}^{\text {Rot }}\right)$ and CGC geometry $\left(\rho_{3}^{\text {Rot }}\right)$ in their respective rotated frames for $I_{1}-I_{4}$ (from bottom up). Right: Corresponding ratios for $v_{2}$ s from $I_{1}-I_{4}$ (lines) and for the eccentricity (open circles). Note that the CGC geometry has larger eccentricity.
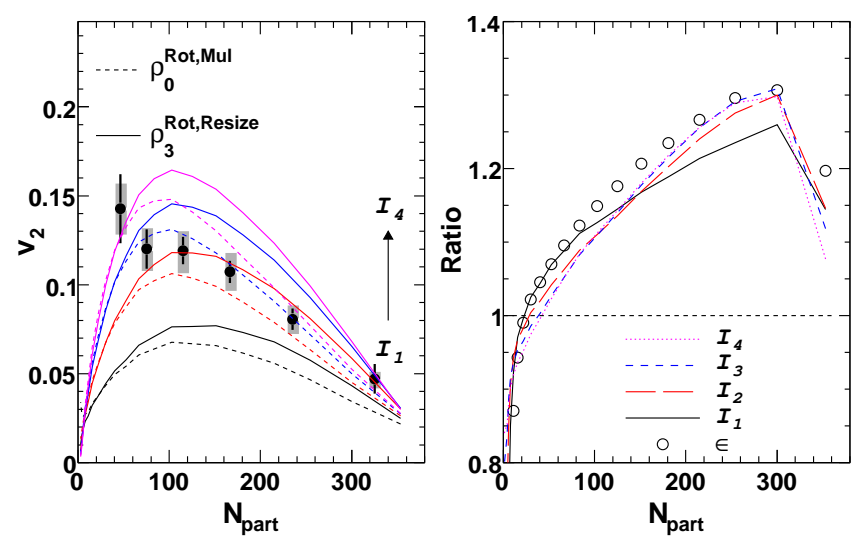

FIG. 8: (Color online) Same as Fig. 7, except that the RMS size of CGC geometry has been stretched to match Glauber geometry $\rho_{0}$ (according to ratio shown in bottom right panel of Fig. 5)

These comparison plots clearly show that both eccentricity and the size contribute to the difference of the $v_{2}$ between CGC and Glauber geometry: While the eccentricity of the CGC geometry increase by about 10\%-30\% relative to Glauber geometry, its transverse size shrinks. The latter change increases the fractional contribution of surface jets, which have smaller $v_{2}$. In contrast, there is no such bias for hydrodynamic calculation of low $p_{T} v_{2}$, which depends on the shape, not the size, of the matter profile.

\section{Glauber geometry based on collision profile and two component profile}

The preceding discussion alludes to an interesting possibility: For a given energy loss formula and jet production profile, as long as the matter profile is adjusted to a common reference $\sigma_{r}$ and $d N / d y$, the $v_{2}$ depends only on the eccentricity of the matter profile. Here we further test this ansatz by using a matter profile $\rho_{1}$ that is very different from $\rho_{0}$. Comparing to $\rho_{0}, \rho_{1}$ has much larger eccentricity (left panels of Fig. 9) which should increase the calculated $v_{2}$. On the one hand, it has stronger centrality dependence of total integral (Fig. 2) and 10\%-15\% smaller $\sigma_{r}$ (right panels of Fig. 9), both are expected to significantly decrease the calculated $v_{2}$.
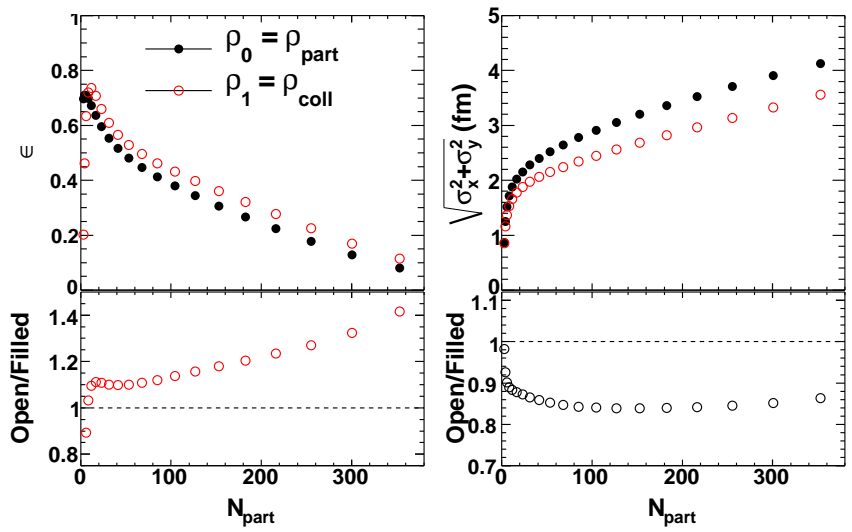

FIG. 9: (Color online) Left: Eccentricities for Glauber geometry calculated from participant density profile $\rho_{0}$ (solid circles) and from collision density profile $\rho_{1}$ (open circles) in the top panel and the corresponding ratio in the bottom panel. Right: Same as right panels except they are for the RMS size $\sigma_{r}=\sqrt{\sigma_{x}^{2}+\sigma_{y}^{2}}$.

Figure 10 compares the $v_{2}$ calculated for $\rho_{1}$ and $\rho_{0}$ in their respective rotated frames. The calculated $v_{2}$ falls well below the experimental $v_{2}$ data in central collisions, which suggests that the Glauber geometry based solely on collision density profile with eccentricity fluctuation is ruled out. The decrease of the $v_{2}$ is largely attributable to the stronger centrality dependence and smaller size of $\rho_{1}$, and can be seen more quantitatively in the right panel, which appears as a large suppression of the $v_{2}$ ratios from the expected eccentricity ratio.

To dissect the impacts of these factors more clearly, we calculate the $v_{2}$ of $\rho_{1}$ in three different ways before making the ratio with the $v_{2}$ of $\rho_{0}^{\text {Rot,Mul }}$ 1) original multi- 
plicity and size, $\left.\rho_{1}^{\text {Rot }} ; 2\right)$ multiplicity is adjusted to match $d N / d y$ or $\left.\rho_{0}^{\text {Rot,Mul }}, \rho_{1}^{\text {Rot,Mul }} ; 3\right)$ both multiplicity and size adjusted to match $\rho_{0}^{\text {Rot,Mul }}, \rho_{1}^{\text {Rot,Mul,Resize }}$. The results are shown in Fig. 11 for $m=1$ (top-left panel), $m=2$ (top-right panel), $m=3$ (bottom-left panel), and $m=4$ (bottom-right panel). Again the $\kappa$ is readjusted independently for each case. We see that matching the multiplicity dependence mostly increases $v_{2}$ at $N_{\text {part }}<200$ where the $d N / d y$ per participant is changing the fastest, but has very little influence at $N_{\text {part }}>200$. However, after the RMS size of the matter profile is readjusted to match that of the participant profile, the calculated $v_{2}$ ratios follow the ratio of the eccentricities nicely (except in central and peripheral bins for $m>1)$. We clearly see a large sensitivity of $v_{2}$ on $\sigma_{r}$ : The large $(\sim 50 \%)$ suppression of $v_{2}$ in central collisions, that is, the difference between the dashed line and the solid circles, is mostly attributable to a $\sim 15 \%$ narrowing of $\sigma_{r}$ (Fig. 9).
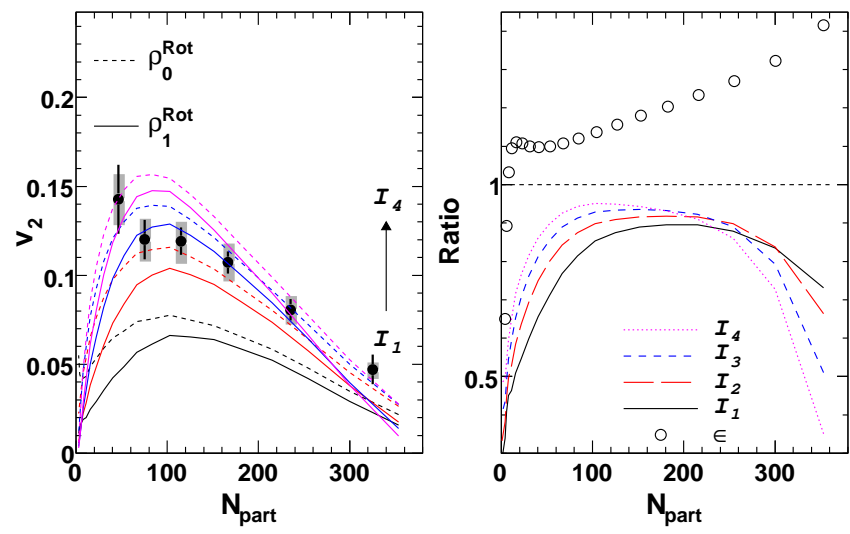

FIG. 10: (Color online) Left: $v_{2}$ calculated for two Glauber geometries, $\rho_{0}^{\text {Rot }}$ and $\rho_{1}^{\text {Rot }}$, in their respective rotated frames for $I_{1}-I_{4}$ (from bottom up). Right: Corresponding ratios for $v_{2} \mathrm{~s}$ from $I_{1}-I_{4}$ (lines) and for the eccentricity (open circles). Note that $\rho_{1}^{\text {Rot }}$ (collision density profile) has larger eccentricity.

It is now straightforward to apply what we learned from Fig. [11 to study the $v_{2}$ for the two component matter profile, $\rho_{2} . \rho_{2}$ is built as a linear combination of participant density profile $\left(\rho_{1}\right)$ and collision density profile $\left(\rho_{0}\right)$. It is a quite popular initial geometry used in many hydrodynamic model calculations [30, 39, 40]. It has the correct multiplicity; but a smaller geometrical size relative to $\rho_{0}$ due to a centrality-dependent contribution from binary collision density profile (Even though $\delta$ is fixed at $0.14, \rho_{\text {coll }}$ becomes more important in central collisions because $N_{\text {coll }}$ grows faster than $N_{\text {part }}$ toward central collisions.). We naturally expect the corresponding eccentricity and $v_{2}$ should sit between that for $\rho_{0}$ and $\rho_{1}$. This is indeed the case as shown by Fig. 12, The ratio of the calculated $v_{2}$ between $\rho_{2}$ and $\rho_{0}$ has shape similar to that of the middle curve in Fig. 11, albeit the rate of change is reduced. The $v_{2}$ ratios decrease with $N_{\text {part }}$, while the ratio of eccentricity increases slightly with $N_{\text {part }}$. They

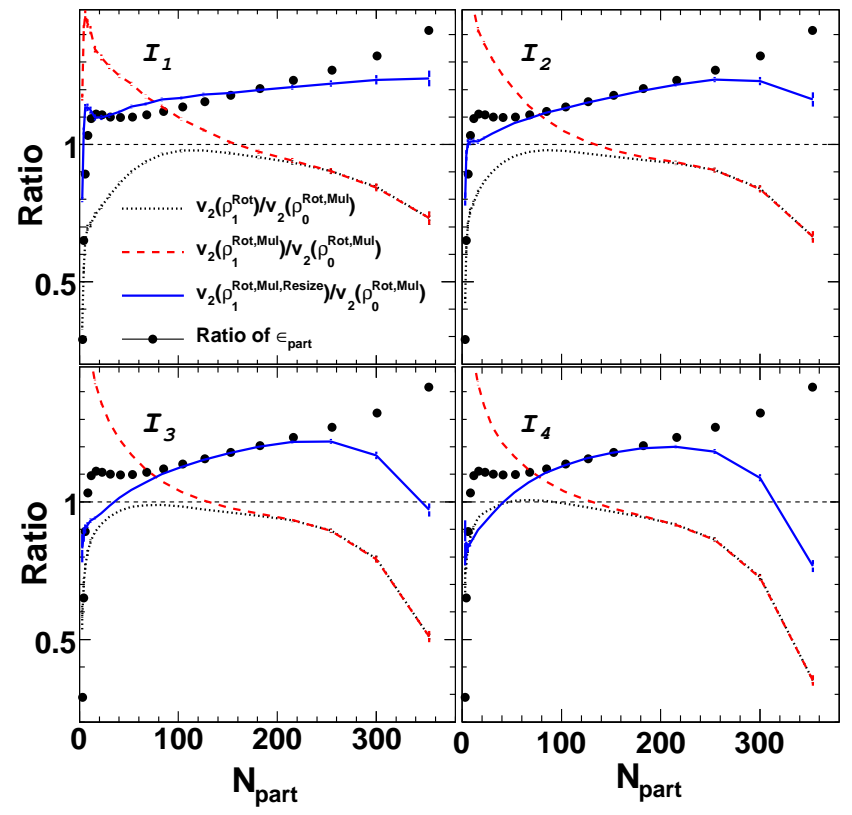

FIG. 11: (Color online) Ratios of calculated $v_{2}$ between $\rho_{0}^{\text {Rot,Mul }}$ and three cases of $\rho_{1}^{\text {Rot }}$ : original multiplicity and size (dotted lines), multiplicity is adjusted to match $d N / d y$ or $\rho_{0}^{\text {Rot, Mul }}$ (dashed lines), both multiplicity and size adjusted to match $\rho_{0}^{\text {Rot, Mul }}$ (solid lines). They are presented separately for $I_{1}$ (top left panel), $I_{2}$ (top right panel), $I_{3}$ (bottom left panel) and $I_{4}$ (bottom right panel).

cross each other at around $N_{\text {part }} \sim 100$. In most central collisions the $v_{2}$ from two component model is suppressed by about $10 \%$, even though the eccentricity value shows $\sim 10 \%$ increase. Consequently, it under-predicts the data in central collisions (see left panel).
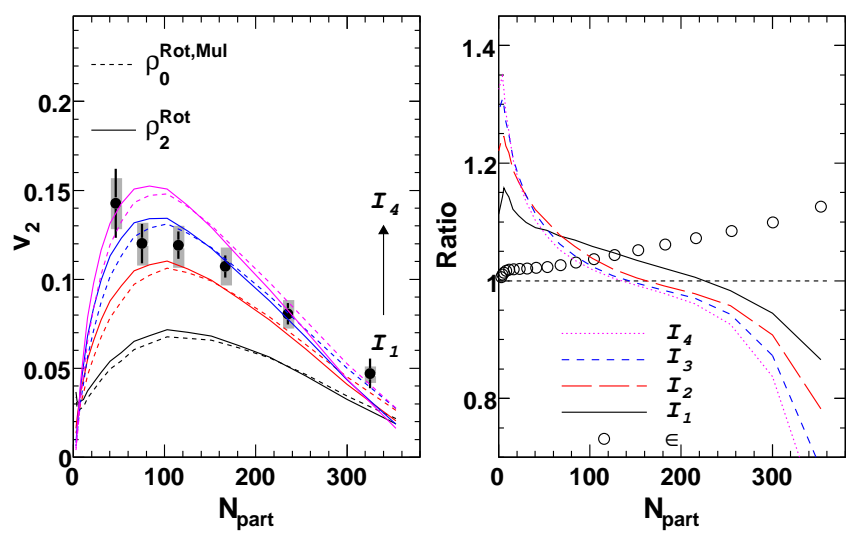

FIG. 12: (Color online) Left: $v_{2}$ calculated for two Glauber geometries, $\rho_{0}^{\text {Rot }}$ and $\rho_{2}^{\text {Rot }}$, in their respective rotated frames for $I_{1}-I_{4}$ (from bottom up). Right: Corresponding ratios for $v_{2}$ s from $I_{1}-I_{4}$ (lines) and for the eccentricity (open circles). Note that $\rho_{2}^{\text {Rot }}$ (two component model density profile) has larger eccentricity.

As a final note, we point out that the mixing fraction, 
$\delta=0.14$, between $N_{\text {part }}$ and $N_{\text {coll }}$ is chosen to match the $d N / d y$. However, it is not clear that this parametrization necessarily reflects the true shape and size of the matter profile (see for example [50]). This concern is especially true in viewing its poor agreement with the central data for $I_{1}-I_{4}$ even after including the eccentricity fluctuation. Such poor agreement is clearly due to the narrow profile of the collision component in the two component profile. Nevertheless, it seems that by combining the hydrodynamic description of the low $p_{T} v_{2}$ and the jet quenching description of the high $p_{T} v_{2}$, one can gain insight not only on the eccentricity or shape, but also the size of the matter profile.

\section{Fluctuations beyond rotation to the participant plane}

The study of fluctuation so far only includes the fluctuation of PP angle of the initial geometry. In principle, we should also consider local density fluctuations which affect variance along the long and short axes of the ellipsoid without changing the orientation of the PP. In other words, both the size $\sigma_{r}$ and the participant eccentricity $\epsilon_{\mathrm{part}}$, which are invariant under rotation, can still fluctuate event to event for fixed impact parameter. These fluctuations are large compared to their mean values, as shown by Fig. 13.

Estimation of the influence of these additional fluctuations requires event-by-event calculation of the jet absorption, where the nucleons cannot be treated as pointlike objects. We assume the nucleon has a Gaussian profile in the transverse plane with a width of $r_{0}$ in the $x$ and $y$ directions, corresponding to a nucleon-nucleon overlap function,

$$
t(x, y)=\frac{1}{2 \pi r_{0}^{2}} e^{-\frac{x^{2}+y^{2}}{2 r_{0}^{2}}},
$$

and a binary collision profile,

$$
\begin{aligned}
\rho_{\text {coll }}(x, y, b)=\int d x^{\prime} d y^{\prime} & T_{A}\left(x-\frac{b}{2}, y\right) \times \\
& T_{A}\left(x+\frac{b}{2}+x^{\prime}, y+y^{\prime}\right) t\left(x^{\prime}, y^{\prime}\right)
\end{aligned}
$$

The event-by-event participant profile is obtained by summing over the nucleon profile for all participants.

For each event, we generate four dijet pairs by sampling its collision profile [Eq.88, then calculate their absorption in corresponding participant density profile. A total of $3 \times 10^{6}$ Glauber events are used. The $\kappa$ is chosen such that the overall survival rate averaged over all events is 0.18 for the $0 \%-5 \%$ centrality bin. To check the stability of our result against the finite size assumption of the nucleons, we varied the $r_{0}$ from $0.2-0.4 \mathrm{fm}$, and we also assume the nucleon to be disk of constant density with a radius of $\sqrt{\sigma_{\mathrm{nn}}^{\text {inel }} / \pi} / 2=0.58 \mathrm{fm}$. It turns out the final results are not sensitive to details of the nucleon overlap function, except for very peripheral collisions $\left(N_{\text {part }}<\right.$ 20 ) when nucleon size become comparable to the size of the ellipsoid. The deviation is even smaller, when average collision geometry is used. More detailed discussion on this can be found in Appendix C

Figure 14 shows the influences of these additional fluctuations on jet quenching $v_{2}$. Again, the jet absorption strength is tuned independently to match the central $R_{\mathrm{AA}}$ data. The main effect of these additional fluctuations is a small increase of the $v_{2}$ in central collisions and a small decrease in peripheral collisions. The change is less than $10 \%$ for $N_{\text {part }}>100$.

We can understand this $10 \%$ centrality dependence change of the jet quenching $v_{2}$ based on what was learned from previous discussion as follows. First, we notice that the eccentricity averaged over many events is not the same as the eccentricity of the matter profile averaged over many events, that is, $\left\langle\frac{\sigma_{y}^{\prime 2}-\sigma_{x}^{\prime 2}}{\sigma_{y}^{\prime 2}+\sigma_{x}^{\prime 2}}\right\rangle \neq \frac{\left\langle\sigma_{y}^{\prime 2}\right\rangle-\left\langle\sigma_{x}^{\prime 2}\right\rangle}{\left\langle\sigma_{y}^{\prime 2}\right\rangle+\left\langle\sigma_{x}^{\prime 2}\right\rangle}$. However, Fig 27 in Appendix C shows that the difference is only about $2 \%$ and independent of $N_{\text {part }}$ and cannot explain the difference of the $v_{2}$ in Fig. 14. Thus, it must be related to the event-by-event fluctuation of the $\sigma_{r}$. What really matters is the relative size between the jet profile and the matter profile, $R=\sigma_{r}^{\text {coll }} / \sigma_{r}^{\text {part }}$. Smaller $R$ implies that more jets are produced in the interior of the matter profile, thus suffer more energy loss and have large $v_{2}$; larger $R$ implies more jets are produced in the corona region of the matter profile, thus suffer less energy loss and have smaller $v_{2}$.

Figure 15 shows the distribution of $R$ for several centralities, as well as its mean value $\langle R\rangle$ and standard deviation $\sigma_{R}$ as a function of centrality. $\langle R\rangle$ is almost constant as a function of $N_{\text {part }}$ around $\sim 0.85$. However the width of the distribution $\sigma_{R}$ is a strong function of centrality; it increases from about $2 \%$ in most central collisions to more than $5 \%$ around $N_{\text {part }} \sim 50$, and the distribution becomes asymmetric toward peripheral bins. It is true that the initial jet production rate does not depend on the fluctuation of $R$. However, the survival probability does; that is, more jets escape the medium when $R$ fluctuates to large values while fewer jets escape when $R$ fluctuates to small values. Thus this $R$ dependent survival probability amplifies the upward fluctuation of $R$, leading to a smaller $v_{2}$. The suppression of the $v_{2}$ is greater in peripheral collisions due to a broader $R$ distribution. This explains the falling of the ratio toward peripheral collisions in Fig. 14.

Before closing this section, we stress that the effect of fluctuation on $v_{2}$ can be largely attributed to the fluctuation of the PP angle. The residual effects, arising mainly from event-by-event fluctuation of the relative size between matter profile and jet production profile, are less than $10 \%$ for $N_{\text {part }}>100$. Thus, it seems reasonable to use an averaged matter profile and binary collision profile for jet quenching calculation, supplemented with a small centrality dependent correction. This is of prac- 

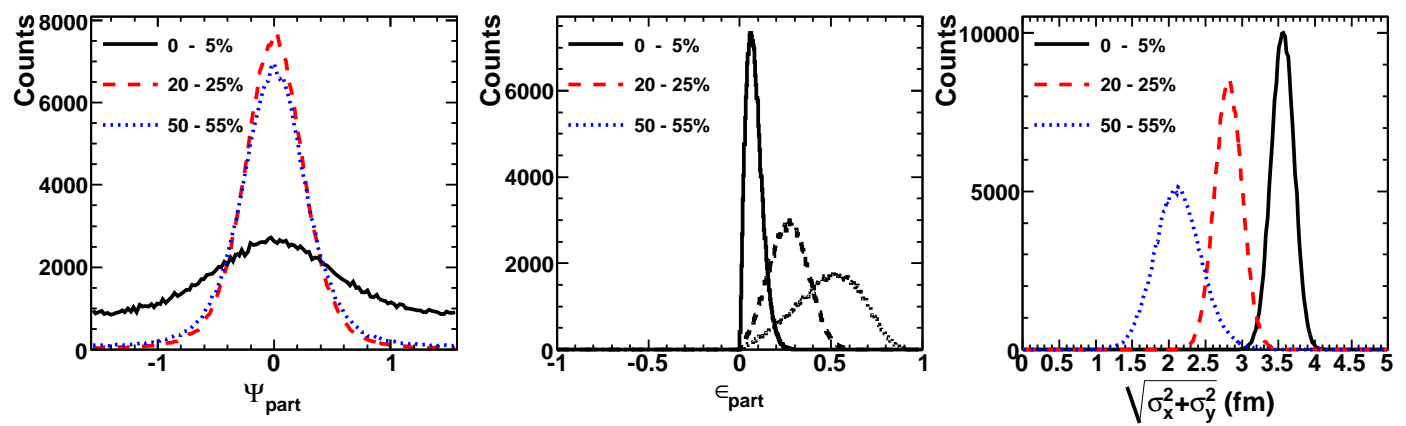

FIG. 13: (Color online) Distributions of participant plane angle (left panel), participant eccentricity (middle panel) and RMS size (right panel) for $\rho_{0}$ in three centrality selections (0\%-5\%, 20\%-25\% and 50\%-55\%).
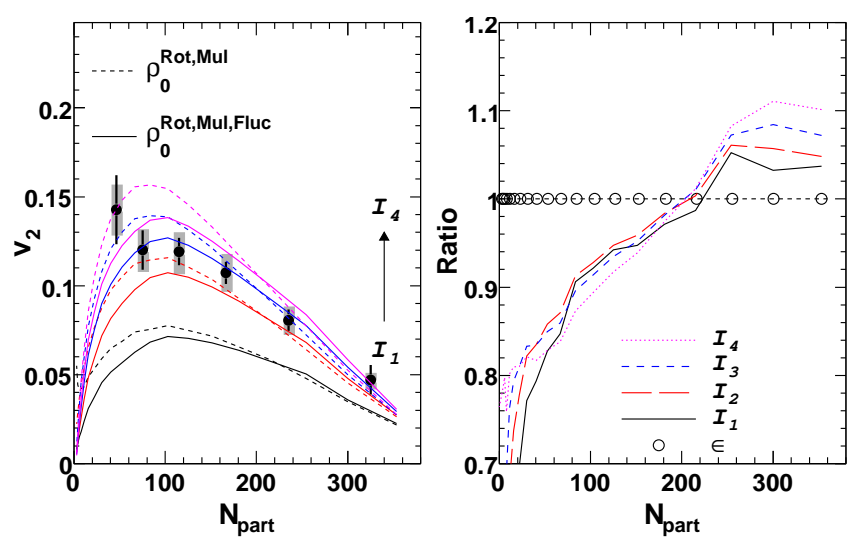

FIG. 14: (Color online) Left: $v_{2}$ for participant density profile (but in rotated frame and scaled to $d N / d y$ ), calculated either on averaged profile $\left(\rho_{0}^{\text {Rot,Mul }}\right)$ or event-by-event with additional fluctuations ( $\left.\rho_{0}^{\text {Rot,Mul,Fluc }}\right)$. Right: Corresponding ratios for $v_{2}$ s from $I_{1}-I_{4}$ (lines) and for the eccentricity (open circles). Note that the two cases have the same eccentricities.

tical importance, because event-by-event jet quenching calculation is either not possible or computationally prohibitive for many current pQCD models. However, the lumpiness for event-by-event geometry implies large fluctuation of scatter centers along the jet trajectory, which may influence the LPM [51] coherent effect; there is no such problem when the event average density profile is used (see Fig. 28, compare the first three panels with the bottom-right panel). However, investigation of such effects is beyond the scope of this study.

\section{E. Dependence on the thermalization time}

One of the main uncertainties in hydrodynamic description of the elliptic flow arises from modeling of the thermalization time $\tau_{0}$, i.e. the time at which the system reaches local equilibrium and hydrodynamic expansion is turned on. This time also explicitly enters the energy loss calculation. The value of $\tau_{0}$ is not known due to lack of constraints on the initial geometry and early time dy- namics. Early estimation based on ideal hydrodynamics and Glauber geometry [52] shows that the RHIC $v_{2}$ data require $\tau_{0}<0.6 \mathrm{fm} / c$ when assuming free-streaming of partons at $\tau<\tau_{0}$. However, Luzum and Romatchke [54] argue that the large initial eccentricity of CGC geometry allows a bigger $\tau_{0}$ (up to $1.5 \mathrm{fm} / c$ ) for free-streaming, without destroying the agreement of their calculation with experimental data. Note that free-streaming is an extreme assumption, since partons always interact with each other and build up flow even if the matter is not in local thermal equilibrium [55]. This is especially true for jet energy loss which does not explicitly require local equilibrium. Nevertheless, it is an interesting question to ask whether the high $p_{T}$ anisotropy due to jet quenching can provide any constraints on $\tau_{0}$.

Current implementations of the pre-equilibrium energy loss are different among various $\mathrm{pQCD}$ models. The value of $\tau_{0}$ typically varies in $0-0.6 \mathrm{fm} / c$. Some calculations assume $\hat{q}=0$, while others assume it is constant at $\tau<$ $\tau_{0}[4,[13,56,57]$. Although both can describe the single inclusive suppression, the extracted $\hat{q}$ at $\tau_{0}$ can differ by as much as a factor of two [58]. In this work, we tried three different formalisms to model the pre-equilibrium energy loss.

- Jets propagate freely to $\tau_{0}=l_{0} / c$, then radiative energy loss and LPM interference are switched on:

$$
\begin{aligned}
I_{a} & =\int_{l_{0}}^{\infty} d l\left(l-l_{0}\right) \frac{\rho(\overrightarrow{\mathbf{r}}+l \widehat{\mathbf{v}})}{l} \\
& =\int_{0}^{\infty} d l \frac{l}{l+l_{0}} \rho\left(\overrightarrow{\mathbf{r}}+\left(l+l_{0}\right) \widehat{\mathbf{v}}\right)
\end{aligned}
$$

- LPM effects start at $\tau=0$, but its contribution to energy loss is truncated at $\tau<\tau_{0}$ :

$$
\begin{aligned}
I_{b} & =\int_{0}^{\infty} d l \frac{\rho(\overrightarrow{\mathbf{r}}+l \widehat{\mathbf{v}})}{l} \times \begin{cases}0 & l \leq l_{0} \\
l & l>l_{0}\end{cases} \\
& =\int_{l_{0}}^{\infty} d l \rho(\overrightarrow{\mathbf{r}}+l \widehat{\mathbf{v}})
\end{aligned}
$$

This functional form is motivated by often made claims similar to one from Ref. [13]: "For times 

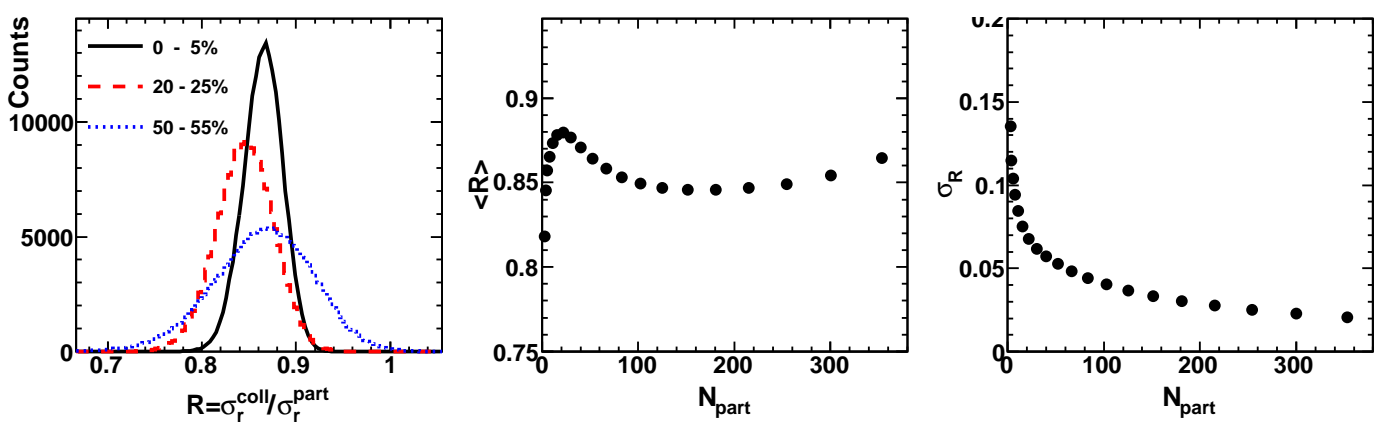

FIG. 15: (Color online) Left: Distributions of event-by-event ratio of RMS width of collision density profile to participant density profile, $R=\sigma_{r}^{\text {coll }} / \sigma_{r}^{\text {part }}$. Middle: centrality dependence of the mean value for $R,\langle R\rangle$. Right: centrality dependence of the RMS width for $R, \sigma_{R}$.

prior to $0.6 \mathrm{fm} / \mathrm{c}$, i.e., the starting point of the RFD simulation, we neglect any medium effects, i.e., assume $\hat{q}=0$. Note that for a purely radiative energy loss model where the average energy loss grows quadratically with path length in a constant medium the effect of initial time dynamics is systematically suppressed and no strong dependence of the energy loss on variations of the initial time is observed."

- Radiative energy loss is on all the time, but the experienced density is assumed to increase linearly with time and reach the local density at $\tau_{0}$.

$$
I_{c}=\int_{0}^{\infty} d l \times \begin{cases}\rho\left(\overrightarrow{\mathbf{r}}+l_{0} \widehat{\mathbf{v}}\right) \frac{l}{l_{0}} & l \leq l_{0} \\ \rho(\overrightarrow{\mathbf{r}}+l \widehat{\mathbf{v}}) & l>l_{0}\end{cases}
$$

This effectively implies a $\hat{q}$ that grows and reach maximum at $\tau_{0}$.

Figure 16 shows the calculation for various values of $\tau_{0}$ from 0 to $2.0 \mathrm{fm} / c$ for the three cases in the rotated frame. We find that $I_{a}$ exhibits the strongest dependence on $\tau_{0}$. The calculated $v_{2}$ increases almost linearly with $\tau_{0}$ and reaches the experimental data at $\tau_{0} \sim 1.5 \mathrm{fm} / c$. This time is somewhat smaller than a similar analysis from Pantuev [59], who need $\tau_{0} \sim 2-3 \mathrm{fm} / c$ to match the data. $\tau_{0}$ is smaller in our case because we include eccentricity fluctuation. The increase of $v_{2}$ with $\tau_{0}$ can be attributed to increasingly larger contribution from jets originated from the corona region of the overlap, whose size is proportional to $\tau_{0}[59]^{3}$. Note that the dependence

3 This can be qualitatively understood as the following. Equation 9 defines a corona region $l<l_{0}\left(I_{a}\right.$ is less suppressed) and a core region $l>l_{0}$ ( $I_{a}$ is more suppressed), where $l$ is the distance from the surface. The radial distribution of the initial positions for the surviving jets is largely defined by the requirement that $R_{A A}=0.18$ or $18 \%$ jets survive in most central collisions. When $l_{0}$ is small, the corona volume is $<18 \%$, the surviving jets come from both the corona and the core and have a quite broad radial distribution. As $l_{0}$ or corona volume grows, more and more surviving jets originate from the corona region with larger of jet quenching $v_{2}$ on $\tau_{0}$ is just the opposite of that for elliptic flow; the latter always decreases with increasing $\tau_{0}$.

For the second functional form, $I_{b}$, we find that the truncation of contribution at $\tau<\tau_{0}$ does simulate the suppression of the early contribution due to quadratic path length dependence. However, $I_{b}$ exhibits a much weaker dependence on $\tau_{0}$ than $I_{a}$, so the two are not equivalent. The increase of $v_{2}$ reaches about $20 \%$ for $\tau_{0}=$ $0.6 \mathrm{fm} / c$ and grows continuously thereafter. It reaches the experimental value at $\tau_{0}=2.0 \mathrm{fm} / c$ instead of $\tau_{0}=$ $1.5 \mathrm{fm} / c$ for $I_{a}$.

As a more realistic scenario that takes into account some contributions from $\tau<\tau_{0}, I_{c}$ exhibits much weaker dependence on $\tau_{0}$. The change in $v_{2}$ is less than $10 \%$ at $\tau_{0}<0.6 \mathrm{fm} / c$; but it again increases quickly at large $\tau_{0}$ ( $>1 \mathrm{fm} / c)$, where matter integral is dominated by the corona region which has a large asymmetry.

A slightly different exploration of effects of early time energy loss has been reported in Ref. [58]. It assumes either a constant $\hat{q}\left[\hat{q}=\hat{q}\left(\tau_{0}\right)\right]$ or a $\hat{q}$ that decreases rapidly to its value at $\tau_{0}\left[\hat{q}=\hat{q}\left(\tau_{0}\right)\left(\frac{\tau_{0}}{\tau}\right)^{3 / 4}\right]$. Both cases imply more pre-equilibrium contribution than $I_{c}$; thus, we expect that they have even weaker dependence on $\tau_{0}$ than on $I_{c}$. Note that it is generally true that calculation which has a smaller $\tau_{0}$ or takes into account the contribution at $\tau<\tau_{0}$ always has smaller $v_{2}$, because the early part of the matter integral tends to be more isotropic.

Our calculation does not take into account the transverse expansion. As pointed out earlier 60, 61], the dependence on $\tau_{0}$ is further suppressed if the radial flow is included. This is because the medium moves outward at speed of $v_{T}$. Jets that are generated behind the fluid cell need to move and catch up with it. Thus, matter integrals decrease more slowly with $\tau_{0}$ than the $1+1 \mathrm{D}$ case. Effectively, the radial flow tends to shrink the black core

anisotropy, and the radial distribution narrows. Until corona volume reaches about 18\%, most surviving jets come from corona and the core becomes almost black. 

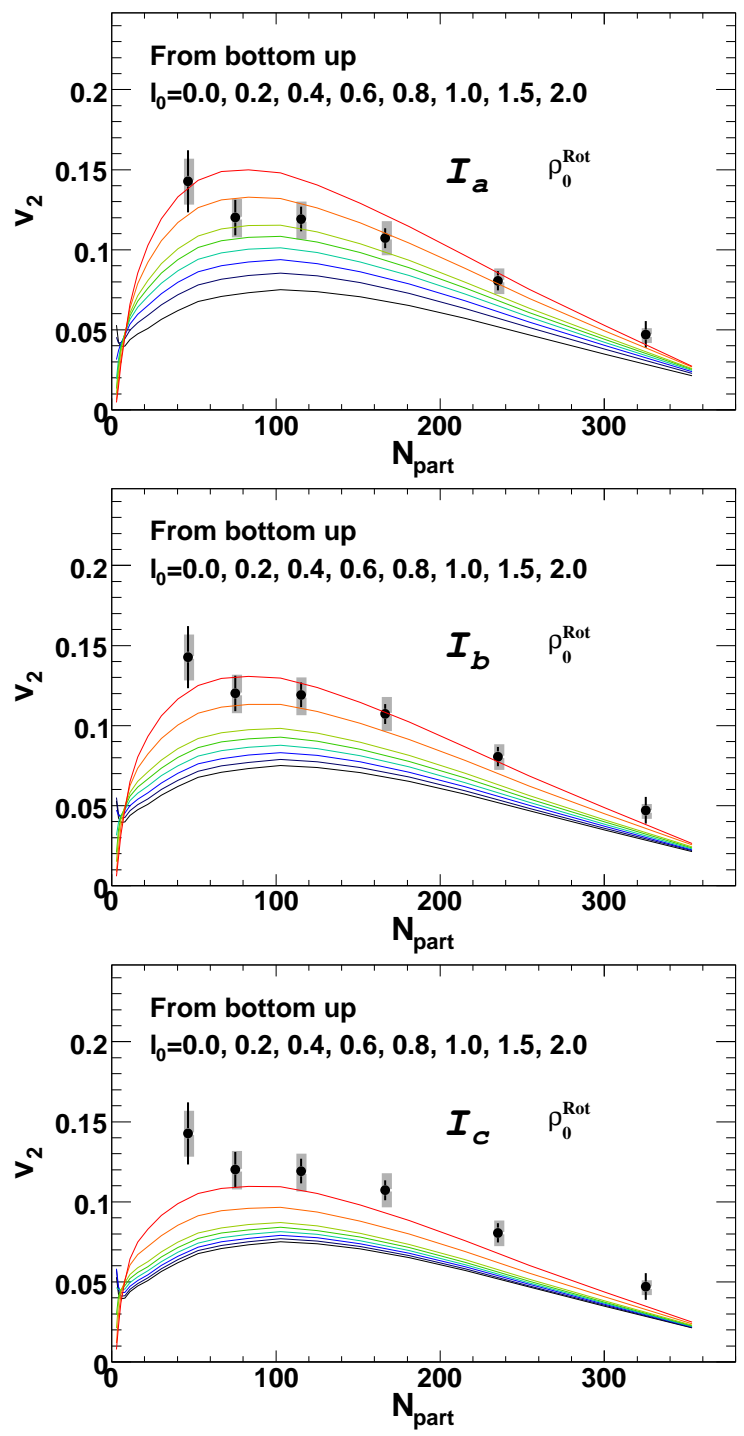

FIG. 16: (Color online) $v_{2}$ calculated using three different schemes for jet absorptions: $I_{a}$ according to Eq.9 (top panel), $I_{b}$ according to Eq. 10 (middle panel), $I_{c}$ according to Eq. 11 (bottom panel). In each cases, 8 different thermalization times range from $l_{0}=0$ to $2 \mathrm{fm}$ are shown (curves that are lower and darker are for smaller $l_{0}$ ).

region and reduce the dependence on $\tau_{0}$.

\section{F. How sensitive is $R_{\mathrm{AA}}$ to initial geometry and energy loss formula?}

The setup of our model framework also provides a convenient way to study the centrality dependence of several other jet quenching observables, such as inclusive single hadron suppression $R_{\mathrm{AA}}$, inclusive away-side per-trigger yield suppression $I_{\mathrm{AA}}$ and its associated anisotropy $v_{2}^{I_{\mathrm{AA}}}$ (i.e. $I_{\mathrm{AA}}$ as function of angle with respect to the PP). It is rather straightforward for us to identify (similar to $v_{2}$ ) the most relevant control factors of the collision geom- etry and path length dependence for these observables. To preserve the flow of the main discussion, we focus this section on the inclusive $R_{\mathrm{AA}}$, because it coupled directly to the $v_{2}$ discussion. We refer the reader to some initial work on $I_{\mathrm{AA}}$ and $v_{2}^{I_{\mathrm{AA}}}$ in Appendix $\mathrm{A}$,

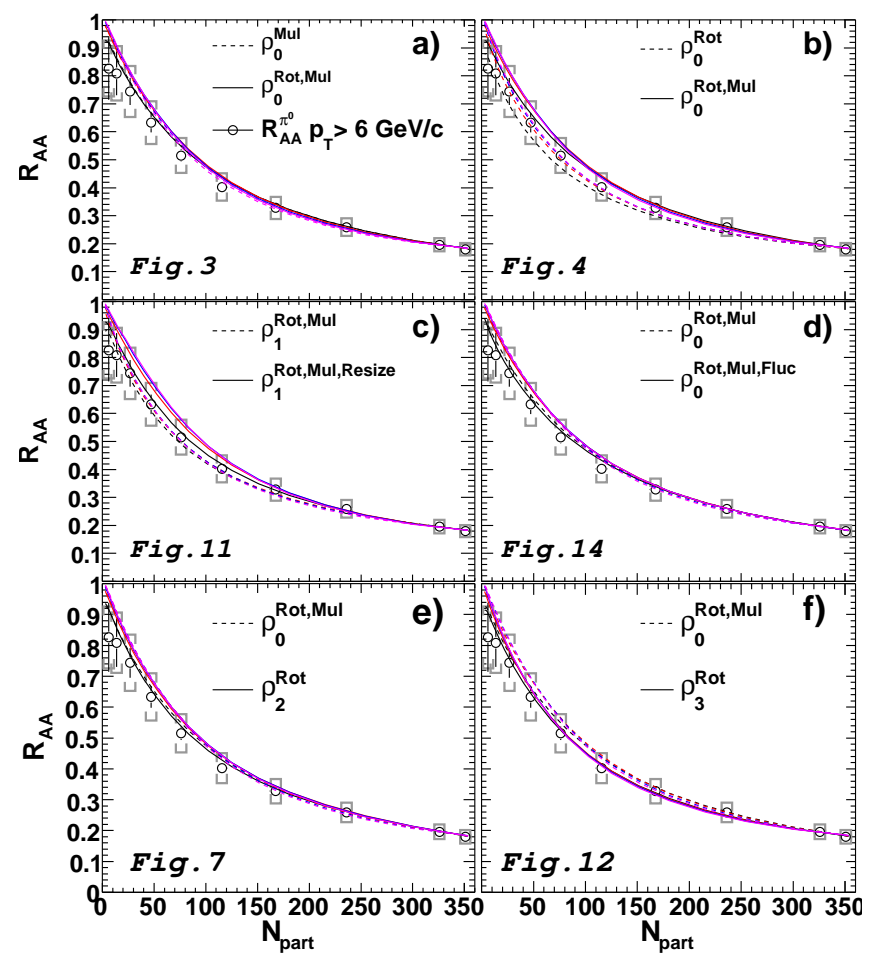

FIG. 17: (Color online) Each of the six panels show the comparison of centrality dependence of $R_{\mathrm{AA}}$ between two matter profiles (matter types are indicated) for $I_{1}-I_{4}$ (eight curves in total). The corresponding $v_{2}$ figure for the same set of geometries is indicated in each panel. The first four panels shows the effects of switching on and off particular effects of the geometry, i.e. Rotation to participant plane for participant profile (panel a)), matching $d N / d y$ for participant profile (panel b)), matching the size for collisional profile (panel c)), and including the additional fluctuation (panel d)). The remaining two panels show comparison between Glauber and CGC geometry (Panel e)) and between two Glauber geometries (Panel f)), respectively.

Figure 17 shows the calculated $R_{\mathrm{AA}}$ in six different cases, with each one designed to check the sensitivity on one aspect of the geometry. From top to bottom and left to right, the lists of checked effects as follows:

a) Without $\left(\rho_{0}^{\text {Rot }}\right)$ and with $\left(\rho_{0}^{\text {Rot,Mul }}\right)$ eccentricity fluctuation. The corresponding $v_{2}$ comparisons are shown in Fig. 3.

b) Without $\left(\rho_{0}^{\text {Rot }}\right)$ and with $\left(\rho_{0}^{\text {Rot,Mul }}\right)$ matching the multiplicity. The corresponding $v_{2}$ comparisons are shown in Fig. 4.

c) Without $\left(\rho_{1}^{\mathrm{Rot}, \mathrm{Mul}}\right)$ and with $\left(\rho_{0}^{\mathrm{Rot}, \mathrm{Mul}, \text { Resize }}\right)$ readjusting the RMS size. The corresponding $v_{2}$ are indicated by the dashed and solid lines in Fig. 11. 
d) Without $\left(\rho_{1}^{\text {Rot,Mul }}\right)$ and with $\left(\rho_{1}^{\text {Rot,Mul,Fluc }}\right)$ additional fluctuation beyond rotation of the PP. The corresponding $v_{2}$ comparisons are shown in Fig. 15.

e) Glauber geometry based on $\left(\rho_{0}^{\text {Rot, Mul }}\right)$ versus CGC geometry $\left(\rho_{3}^{\text {Rot }}\right)$. The corresponding $v_{2}$ comparisons are shown in Fig. 7.

f) Two glauber geometries: participant profile $\left(\rho_{0}^{\text {Rot,Mul }}\right)$ versus two component profile $\left(\rho_{2}^{\text {Rot }}\right)$. The corresponding $v_{2}$ comparisons are shown in Fig. 12.

In each case, all four path-length dependencies $I_{1}-I_{4}$ are shown with and without the particular effect under investigation. Figure 18 compares the different choices of the thermalization time $\tau_{0}$ in a broad range using formulation $I_{a}$ (left) and $I_{c}$ (right); the corresponding $v_{2}$ comparisons are shown in Fig. 16.

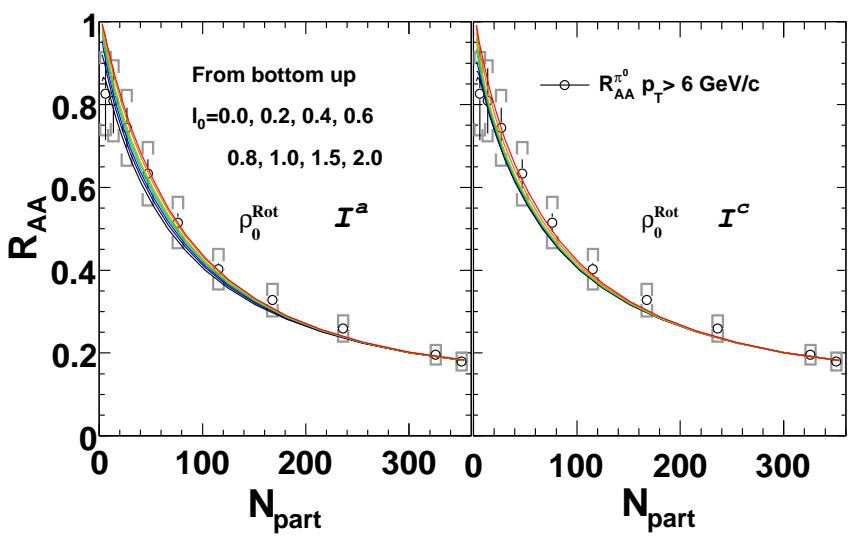

FIG. 18: (Color online) The centrality dependence of $R_{\mathrm{AA}}$ as a function of thermalization time $l_{0} / c$. Two different jet absorption schemes are used, that is, $I_{a}$ in the left panel (Eq. 9 corresponding $v_{2}$ figure is the top panel of Fig. 16) and $I_{c}$ in the right panel (Eq. 11] corresponding $v_{2}$ figure is the top panel of Fig. 16). Curves that are lower and darker corresponds to smaller $l_{0}$.

It appears that when jet absorption strength is tuned to reproduce the suppression in most central collisions, the centrality dependence of $R_{\mathrm{AA}}$ has limited sensitivity on different choices of the collision geometry and energy-loss formula. This confirms previous observations [17, 53] that the centrality dependence of the $R_{\mathrm{AA}}$ has limited discriminating power to dynamics of the underlying energy-loss mechanisms. This is partly due to the energy loss bias, but it is also related to the fact that $R_{\mathrm{AA}}$ has to vary monotonically between $R_{\mathrm{AA}} \sim 0.2$ at large $N_{\text {part }}$ and $R_{\mathrm{AA}} \sim 1$ when $N_{\text {part }} \rightarrow 0$. However, $R_{\mathrm{AA}}$ does exhibit some sensitivities for three cases; that is, it increases somewhat for larger $\tau_{0}$ (Fig. 18), stronger centrality dependence of $d N / d y$ (Fig. 17b) and larger
RMS size of the matter profile (Fig. 17k) ${ }^{4}$. However, the change is well within typical range of the experimental systematic errors. The situation is very different for $v_{2}$ or anisotropy of $R_{\mathrm{AA}}$, which is not a monotonic function of $N_{\text {part }}$, and exhibits a much greater sensitivity to the variation of geometry and energy-loss scheme.

\section{DISCUSSION AND SUMMARY}

Table I summarizes the sensitivity of $v_{2}$ and $R_{\mathrm{AA}}$ on the choices of matter profile and energy-loss formula (also on experimental uncertainties; see Appendix (B). The former includes fluctuation and shape distortion, which affect the eccentricity, centrality dependence of multiplicity, and the size of the matter profile. The latter includes different choices of path length dependence and thermalization time. Overall, $R_{\mathrm{AA}}$ is not sensitive to these factors, while $v_{2}$ has fairly strong dependence on both initial geometry and energy loss formula. We can summarize the main findings for $v_{2}$ as follows.

- Eccentricity fluctuation has to be included in the jet quenching calculation. Without it one can not account for the large $v_{2}$ observed in central collisions. We estimate that it leads to $15 \%-20 \%$ increase of the $v_{2}$ in the mid-centrality bin (Fig. 3).

- The residual event-by-event fluctuation, other than from eccentricity fluctuation, at most leads to $4 \%$ $8 \%$ change in $v_{2}$ at $N_{\text {part }}>100$ (Fig. 14).

- A reasonable variation of the multiplicity dependence, only significantly changes the $v_{2}$ at $N_{\text {part }}<$ 100 (Fig. 4).

- CGC geometry always results in a larger $v_{2}$ than Glauber geometry at $N_{\text {part }}>150$. Depending on the choices of Glauber geometry, the increase ranges anywhere from $10 \%\left(\rho_{\text {part }}\right.$, Fig. 7$), 30 \%$ (two component profile $\rho_{2}$, Fig. 12), or $20 \%$ to $>50 \%$ ( $\rho_{\text {coll }}$, Fig. 11).

- $v_{2}$ is very sensitive to change of the RMS width $\sigma_{r}$ of the profile. A $15 \%$ change in $\sigma_{r}$ can lead to about 30\%-40\% change in calculated $v_{2}$ (Fig. 11).

These same conclusions seem also to apply for the anisotropy of away-side suppression $v_{2}^{I_{\mathrm{AA}}}$ (see Fig. 21), except that the sensitives seem to be much stronger. We

\footnotetext{
${ }^{4}$ Naively, one would expect the $R_{\mathrm{AA}}$ to decrease for larger $\sigma_{r}$ because more jets originate from inside the profile. However, this is only true if jet absorption strength $\kappa$ remains the same. Because we always readjust $\kappa$ such that $R_{\mathrm{AA}} \sim 0.18$ in the most central bin, it almost cancels the expected suppression in large $N_{\text {part }}$ and even makes the peripheral bin less suppressed (Fig. 17f). This is very different from $v_{2}$, which always increases for larger $\sigma_{r}$ (see Fig. 11).
} 
TABLE I: The sensitivity of the $v_{2}$ and $R_{\mathrm{AA}}$ for various changes in matter profile (relative to participant profile) and energy loss schemes.

\begin{tabular}{|c|c|c|}
\hline Types of changes & $v_{2}$ & $R_{\mathrm{AA}}$ \\
\hline Fluctuation of RP angle & $\begin{array}{l}\text { Increase by } 15 \%-30 \% \text { for mid-centrality, much larger } \\
\text { in central and peripheral (Fig. 3) }\end{array}$ & $<5 \%$ \\
\hline $\begin{array}{l}\text { Additional fluctuation } \\
\text { (relevant for e-b-e jet } \\
\text { quenching calculation) } \\
\end{array}$ & $\pm 5 \%-10 \%$ (Fig. 14) & $<5 \%$ \\
\hline Change in average shape & $\begin{array}{l}\text { Increase by } 15 \%-30 \% \text { for CGC (Fig. } 7 \text { and } 8 \text { ), by } 10 \% \\
\text { for two component model (Fig. 12), } 10 \%-40 \% \text { for } \rho_{\text {coll }} \\
\text { profile (Fig. 11) }\end{array}$ & $<5 \%$ \\
\hline $\begin{array}{l}\text { RMS size of matter profile } \\
\left(\sigma_{r}\right)\end{array}$ & $\begin{array}{l}0 \% \text { to }-10 \% \text { change for CGC (compare Fig. } 7 \text { to } 8 \text { ), } \\
\sim 10 \% \text { to }-40 \% \text { change for } \rho_{\text {coll }} \text { (Fig. 11, compare } \\
\text { dashed to solid line) }\end{array}$ & $\begin{array}{l}10 \% \text { change for } 10 \% \\
\text { difference }\end{array}$ \\
\hline $\begin{array}{l}\text { Centrality dependence of } \\
\text { multiplicity }\end{array}$ & Important for $N_{\text {part }}<150$ (Fig. (4) & $\begin{array}{l}10 \%-20 \% \text { for } \\
\text { mid-centrality }\end{array}$ \\
\hline Power $n$ for " $l$ " dependence & Very sensitive & $<5 \%$ \\
\hline Thermalization time $\tau_{0}$ & $\begin{array}{l}\text { Sensitive but depends on modeling of energy loss at } \\
\tau<\tau_{0} \text {. For } \tau_{0}=0.6 \mathrm{fm} / c \text {, it increases by } \sim 30 \% \text { for } \\
I_{a},<15 \% \text { for } I_{b} \text { and }<5 \% \text { for } I_{c} \text {. (see Fig. [16) }\end{array}$ & $\begin{array}{l}\text { No, except for } \\
N_{\text {part }}<100\end{array}$ \\
\hline $\begin{array}{l}12 \% \text { variation for } R_{\mathrm{AA}} \text { in } \\
0 \%-5 \% \text { central bin }\end{array}$ & $<7 \%$ at $N_{\text {part }}>100$ (Fig. 24) & $<12 \%$ \\
\hline
\end{tabular}

stress that the dependence of jet quenching $v_{2}$ on geometry is different from that for the low $p_{T} v_{2}$ driven by collective expansion. The latter is sensitive only to the eccentricity of matter profile (items 1 and 3 in Table \).

Despite the rather complicated dependence on the initial geometry for jet quenching $v_{2}$, most of them, such as the fluctuation and distortion due to saturation, can be constrained independently by elliptic flow data [30, 54]. Jet quenching $v_{2}$ appears to be rather sensitive to the choices of energy-loss formula, thus making it an ideal observable for gaining insights on energy-loss mechanisms. Based on the comparisons shown in Fig. 3 and 7, it seems that naive path length dependence motivated by radiative energy loss, $I_{1}$, is insufficient to describe the data for both Glauber and CGC geometry even with eccentricity fluctuations taken into account. It appears that either $2<m<3$ for Glauber geometry based on participant profile or $m \sim 2$ for CGC geometry, both with eccentricity fluctuations, have the best match with the data. Note that $m=2$ corresponds to the AdS/CFT type of energy loss $\Delta E \propto l^{3}$ for a strongly coupled plasma [22]. Similar strong path length dependence is also observed for away-side suppression $I_{\mathrm{AA}}$ and it's anisotropous $v_{2}^{I_{\mathrm{AA}}}$ (see Fig. 20 and 21).

The jet quenching $v_{2}$ is also quite sensitive to thermalization time $\tau_{0}$, but the sensitivity depends on the model- ing of the pre-equilibrium energy loss. By assuming freestreaming up to $\tau_{0}=1.5 \mathrm{fm} / c$ and including eccentricity fluctuation, we can reproduce the experimental data with quadratic path-length dependence of energy loss. However, inclusion of a very modest pre-equilibrium energy loss, for example, $I_{c}$ which assumes a $\hat{q}$ which linearly grow to $\hat{q}\left(\tau_{0}\right)$ at $\tau_{0}$, already significantly suppressed the dependence on $\tau_{0}$ up to $1 \mathrm{fm} / c$. We can draw similar conclusions for away-side suppression $I_{\mathrm{AA}}$ and the associated anisotropy $v_{2}^{I_{\mathrm{AA}}}$ (see Figs. 22 and 23).

The preceding discussion attested to the value of jet quenching $v_{2}$ in understanding the roles of various geometry factors and constraining the energy loss mechanisms. One can obtain more discriminating power by combining all jet quenching observables, $v_{2}, R_{\mathrm{AA}}, I_{\mathrm{AA}}$, and $v_{2}^{I_{\mathrm{AA}}}$ (see Appendix $\mathrm{A}$ for more discussions). Initial theoretical work already demonstrated the value of combining the $R_{\mathrm{AA}}$ and $I_{\mathrm{AA}}$ [8, 58]; One can do a better job by also including a calculation of $v_{2}$. It is worth pointing out that the study of high $p_{T} v_{2}$ benefited significantly from extensive experimental and theoretical work on the low $p_{T} v_{2}$, which has provided important constraints on the initial eccentricity. We have shown that the reverse is also true; i.e. jet quenching observables can also provide useful new insights on the initial geometry, which could help the interpretation of the elliptic flow data. 
In summary, using a simple jet absorption framework, we studied the sensitivity of jet quenching $v_{2}$ to various aspects of collision geometry and the path-length dependence of energy loss. Besides the eccentricity, we found two other ingredients of the collision geometry, namely, the centrality dependence of the matter integral and the relative size between the matter and the jet profiles, are important for jet quenching $v_{2}$. We compare the calculated $v_{2}$ from both Glauber and CGC geometry with experimental data. A path length dependence stronger than the native $\Delta E \propto l^{2}$ dependence from radiative energy loss, as well as the inclusion of the eccentricity fluctuation, are necessary to reproduce the $v_{2}$ data. A detailed comparison between Glauber and CGC geometry shows that a 15\%-30\% increase of initial eccentricity in CGC only results in half the increase in calculated $v_{2}$ due to a small narrowing of the CGC geometry. This points to an interesting possibility: A large $v_{2}$ can be easily generated if the jet production profile is significantly narrower than the matter profile. This requires a transverse profile distribution that narrows with momentum or Bjorken variable $x=2 p_{T} / \sqrt{s}$. This happens for the nucleon-nucleon collisions [62, 63]; however, we are not aware yet of a physical mechanism to produce a significant narrowing at large $x$ in heavy nuclei.

Our estimations are based on a simple jet absorption framework. Admittedly, it is too simplistic to give direct insight on the dynamics of the energy-loss process. However, it proves to be a useful tool for understanding the centrality dependence of various jet quenching observables, for identifying the most relevant factors in the collision geometry and path-length dependence, as well as for estimating the sign and magnitude of the change as we vary those factors. We have documented all the matter and jet profiles used in this study in Ref. [64]. They can be used as input for future, more-realistic jet quenching calculations.

\section{Acknowledgement}

We thank W. Horowitz for stimulating discussions and a careful proof reading of the manuscript; We appreciate valuable discussions with R. Lacey and U. Heinz; We thank H. J. Drescher, A. Dumitru and Y. Nara for providing the MC-KLN code. This research is supported by NSF under award number PHY-0701487 and PHY1019387.

\section{Appendix A: $I_{\mathrm{AA}}$ and its azimuthal anisotropy?}

Initial geometry should also leave footprints on the azimuthal distribution of the away-side jets. The appropriate observable for this purpose is the anisotropy of the per-trigger yield for the away-side jets, $I_{\mathrm{AA}}\left(\phi_{\text {trig }}-\Psi_{\text {part }}\right)$, which reflects the path length dependence of the energy loss for the away-side jet. Due to the surface bias of the trigger jets, the away-side jets on average have longer path length to traverse; thus, they are expected to exhibit stronger suppression and larger anisotropy. In this work, we calculate the anisotropy coefficient as

$$
v_{2}^{I_{\mathrm{AA}}}=\left\langle I_{\mathrm{AA}} \cos 2\left(\phi_{\text {trig }}-\Psi_{\text {part }}\right)\right\rangle
$$

As a warm-up exercise, Fig. 19] shows the azimuthal dependence of per-trigger yield suppression $I_{\mathrm{AA}}$ in $0 \%-20 \%$ and 20\%-60\% centrality, calculated for $\rho_{0}^{\text {Rot,Mul }}$ profile, i.e. participant density profile in rotated frame and rescaled to match the $d N / d y$ data. As one increase the power $m$ of the path length dependence in $I_{m}, I_{\mathrm{AA}}$ for the $20 \%-60 \%$ bin shows a dramatic decrease in the out-ofplane direction where the path length is large, but only a modest decrease in the in-plane direction where the path length is small. That is because the suppression for a given centrality is largely determined by the typical matter integral $I_{m} \sim\langle L\rangle^{m} /\left\langle L_{0}^{m}\right\rangle^{m}$, which changes more rapidly for larger $m$. Here $L_{0}^{m}$ is some typical length scale fixed in central collisions for $m$. Clearly, the increased sensitivity of large $m$ can generate a large anisotropy, hence large $v_{2}^{I_{\mathrm{AA}}}$. However, the price one has to pay is that it leads to a large suppression in the $0 \%-20 \%$ bin as shown in the left panel of Fig. 19. This is because a large $v_{2}^{I_{\mathrm{AA}}}$ naturally implies a strong suppression in central collisions, as long as the suppression is a monotonic function of the path length.

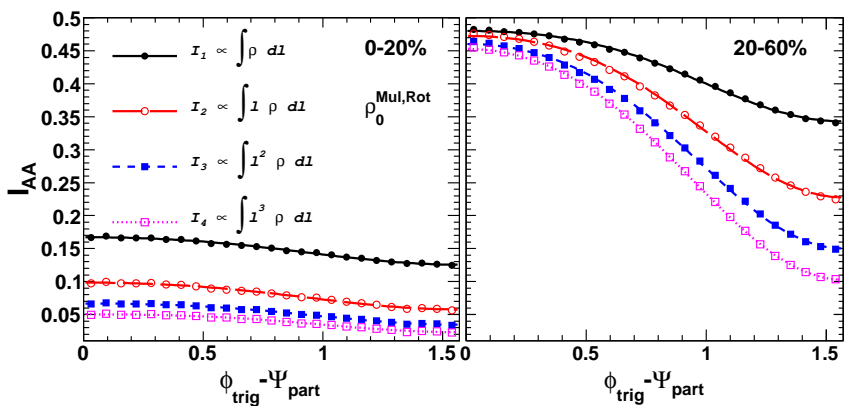

FIG. 19: (Color online) The $I_{\mathrm{AA}}\left(\phi_{\text {trig }}-\Psi_{\text {part }}\right)$ for $0 \%$ $20 \%$ (left panel) and 20\%-60\% (right panel) centrality bins. The points are results of calculation, the line is a fit to $1+2 v_{2}^{I_{\mathrm{AA}}} \cos 2 \Delta \phi+2 v_{4}^{I_{\mathrm{AA}}} \cos 2 \Delta \phi$. We see a finite $v_{4}^{I_{\mathrm{AA}}}$ signal, but it is less than $10 \%$ of $v_{2}^{I_{\mathrm{AA}}}$, and is ignored in this study.

The jet absorption framework used in this work is a pure geometrical model in that the calculated suppression depends only on the path length, thus it always predicts $I_{\mathrm{AA}}<R_{\mathrm{AA}}$ due to the longer path length of the away-side jet. However, experimental data seem to suggest that $I_{\mathrm{AA}} \gtrsim R_{\mathrm{AA}}[7,65,66$. 6 . This is because that the away-side associated hadron spectra are much flatter than the inclusive distribution, due to the requirement of a high $p_{T}$ trigger. For a typical trigger of $5 \mathrm{GeV} / c$, the away-side conditional spectra in $p+p$ collisions at $\sqrt{s}=200 \mathrm{GeV}$, if parameterized via a power law function $1 / p_{T}^{n}$, have a power of $n=8$, in contrast to $n=4$ for 
inclusive hadrons [67]. A simple estimation shows that to reach the same level of suppression, it takes about $50 \%$ more energy loss for away-side jets than for inclusive jets [67]. What this means is that treating energy loss as absorption is not sufficient; an energy shift term is required as well. However, phenomenologically, we can still use the jet absorption framework, if we allow the $\kappa$ to also depend on power $n$; i.e, a smaller $\kappa$ is required for an away-side jet due to a flatter input spectra. Such dependence in principle can be fixed by the $I_{\mathrm{AA}}$ data from STAR and PHENIX [7, 65, 66]. However, we shall defer this improvement to a future study.

Nevertheless, the current setup is sufficient for studying the sensitivity of $I_{\mathrm{AA}}(\Delta \phi)$ on the choices of underlying collision geometry. Figures 20 and 21 summarize the relative change of the inclusive $I_{\mathrm{AA}}$ and its anisotropy $v_{2}^{I_{\mathrm{AA}}}$, respectively, as one varies various aspects of the collision geometry. The six cases in both figures correspond to the same change in collision geometry as in Fig. 17 for inclusive $R_{\mathrm{AA}}$, that is, a) eccentricity fluctuation, $\mathbf{b}$ ) matching the multiplicity, c) re-scaling the geometrical size $\left.\sigma_{r}, \mathbf{d}\right)$ additional fluctuation not included by rotation, f) default Glauber geometry versus CGC geometry, g) default Glauber geometry versus two-component geometry.

In general, we see that $I_{\mathrm{AA}}$ and $v_{2}^{I_{\mathrm{AA}}}$ are much more sensitive to their counterpart single particle observables, $R_{\mathrm{AA}}$ and $v_{2}$. They are also very sensitive to both the path-length dependence and the thermalization time $\tau_{0}$ (see Figs. 222 and 231). In one case (Fig. 21k)), the $v_{2}^{I_{\mathrm{AAA}}}$ even becomes negative in central collisions, reflecting the dominance of tangential emission when the collision profile is used as the matter profile $\left(\rho_{1}\right)$. This is the case because $\rho_{1}$ has the narrowest profile $(15 \%$ smaller than participant profile), such that more jets are generated on the surface and can survive if emitted tangentially. Unlike the $v_{2}$, the change in $v_{2}^{I_{\mathrm{AA}}}$, when tuned to same multiplicity and average, is not proportional to the corresponding change in $\epsilon$. The only change to which $I_{\mathrm{AA}}$ and $v_{2}^{I_{\mathrm{AA}}}$ is not sensitive is the fluctuation of the $\mathrm{PP}$ angle.

\section{Appendix B: Sensitivity to $\kappa$}

We have discussed four physics observables, $R_{\mathrm{AA}}, v_{2}$, $I_{\mathrm{AA}}$, and $v_{2}^{I_{\mathrm{AA}}}$. Their centrality dependencies and path length dependencies are entirely controlled by the $\kappa$ parameter introduced in Sec. [I] For each running mode, $\kappa$ is always readjusted independently such that $R_{\mathrm{AA}}=$ 0.182 in the $0 \%-5 \%$ centrality bin.

The discussion so far has ignored the experimental uncertainty on the $R_{\mathrm{AA}}$ measurement, and it is about $12 \%$ for the $0 \%-5 \%$ centrality bin $\left(0.160<R_{\mathrm{AA}}<0.204\right)$. This uncertainty invariability translates into some uncertainty on $\kappa$, which in turn translates into some uncertainties on the other three observables. Table. [I]summarizes the $\kappa$ values and associated uncertainties calculated for $\rho_{0}, \rho_{2}$ and $\rho_{3}$ and $m=1-4$. One can see that the

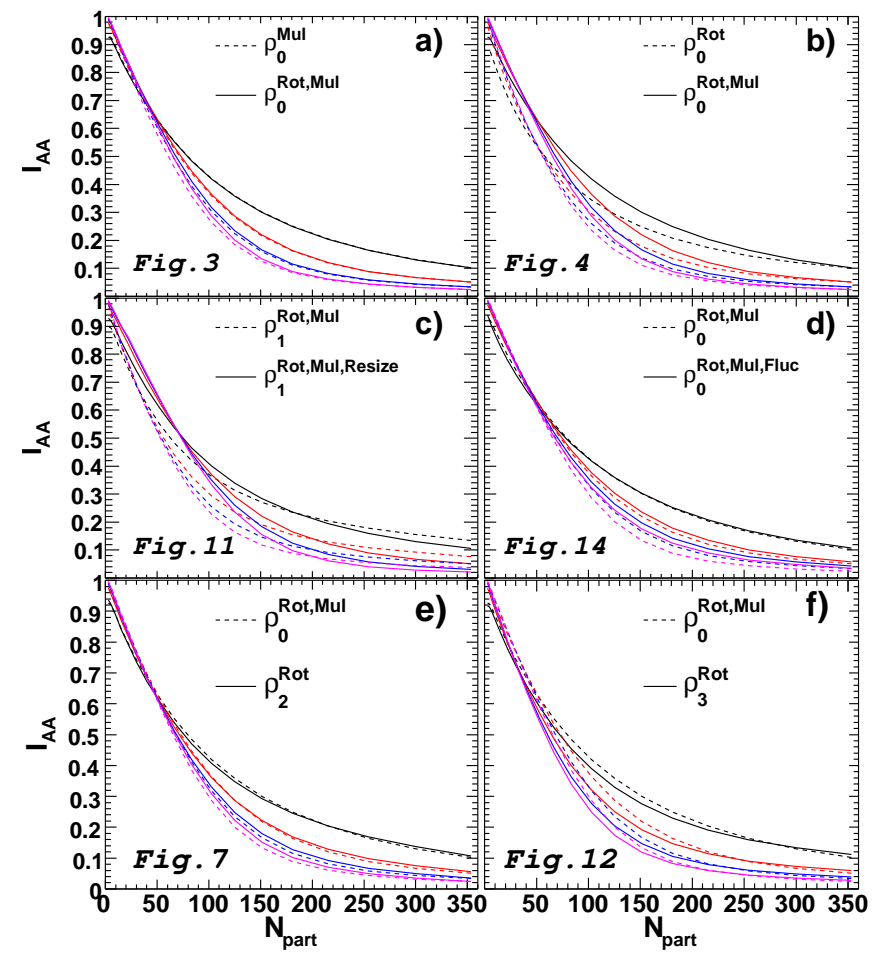

FIG. 20: (Color online) Conventions are similar to those in Fig. 17 Each of the six panels shows the comparison of centrality dependence of $I_{\mathrm{AA}}$ between two matter profiles (matter types are indicated) for $I_{1}-I_{4}$ (eight curves in total); the corresponding $v_{2}$ figures for the same set of geometries are indicated in each panel. The first four panels shows the effects of switching on and off particular effects of the geometry, i.e. rotation of participant plane for participant profile (panel a)), matching $d N / d y$ for participant profile (panel b)), matching the size for collisional profile (panel c)), and including the additional fluctuation (panel d)). The remaining two panels show comparison for Default Glauber vs. CGC geometry (Panel e)) and between two Glauber geometries (Panel f)), respectively. Note that the small difference in panel a) between with and without rotation for $m=4$ should be attributed to increased sensitivity of $I_{\mathrm{AA}}$ to changes in $R_{\mathrm{AA}}$ for large $m$ (because $\kappa$ is tuned by hand to match $R_{\mathrm{AA}} \sim 0.18$ only to the third digit after 0 ).

fractional uncertainties increase gradually for larger $m$. The differences between $\rho_{0}, \rho_{2}$ and $\rho_{3}$, however, can be largely attributed to their different total matter integral in the 0\%-5\% bin, which are 353, 301 and 695 for $\rho_{0}, \rho_{2}$ and $\rho_{3}$, respectively. If we readjust the total matter integral for the $0 \%-5 \%$ centrality bin to the same number, say 353 , then the obtained $\kappa$ would need to be multiplied by $301 / 353=0.854$ for $\rho_{2}$ and $695 / 353=1.97$ for $\rho_{3}$, respectively. This procedure makes the $\kappa$ rather close to each other for different $\rho$ 's; the residual differences then can be attributed to their different shapes and sizes.

Figure 24 shows the centrality dependence of the four 


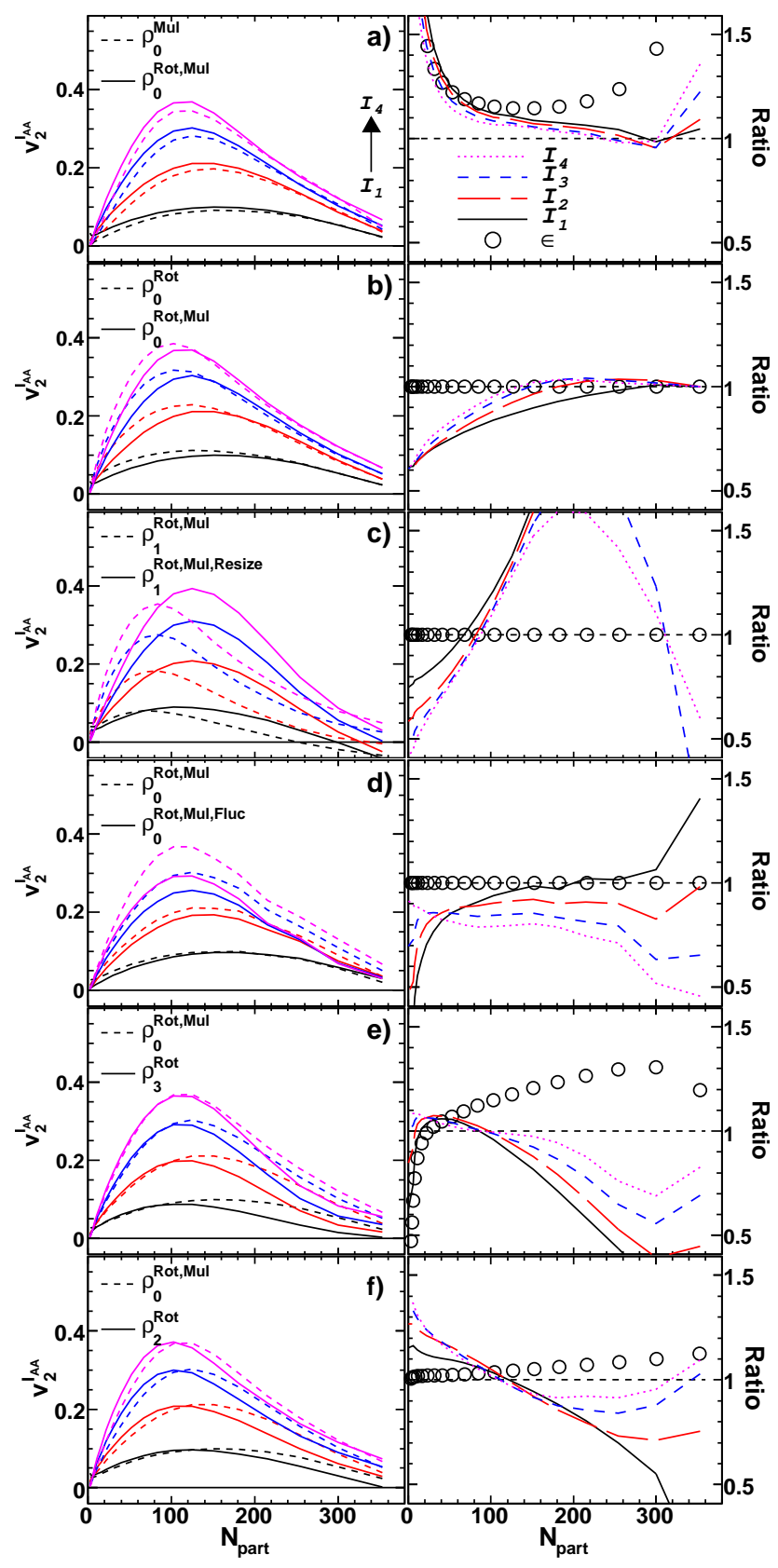

FIG. 21: (Color online) Conventions are similar to those in Fig. 17 Each of the six rows show: (left panel) the comparison of centrality dependence of $v_{2}^{I_{\mathrm{AA}}}$ between two matter profiles (matter types are indicated), (right panel) The corresponding ratios together with ratios of eccentricities. The corresponding $v_{2}^{I_{\mathrm{AA}}}$ plot for the same set of geometries is ndicated in each panel. The first four rows show the effects of switching on and off particular effects of the geometry, i.e. Rotation to participant plane for participant profile (Row a)), matching $d N / d y$ for participant profile (Row b)), matching the size for collisional profile (Row c)), and including the additional fluctuation (Row d)). The remaining two rows show comparison for Default Glauber vs. CGC geometry (Row e)) and between two Glauber geometries (Row f)), respectively.

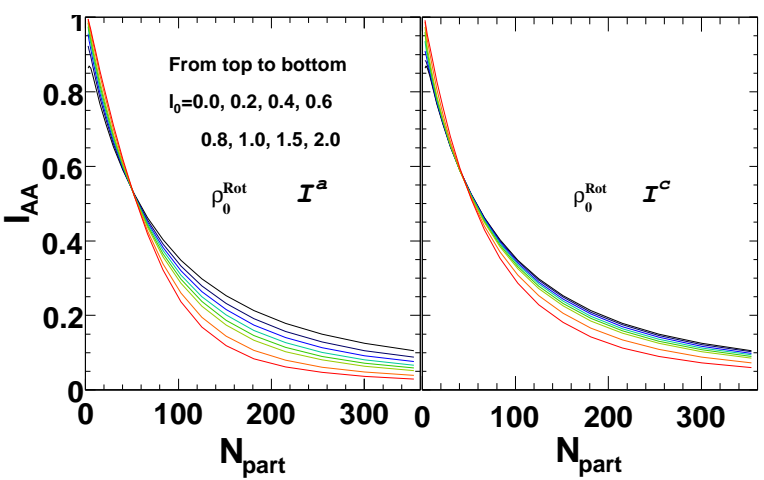

FIG. 22: (Color online) The centrality dependence of $I_{\mathrm{AA}}$ as a function of thermalization time $l_{0}$; Two different jet absorption schemes are used, i.e. $I_{a}$ in the left panel (Eq. 9) and $I_{c}$ in the right panel (Eq. 11). Curves that are higher and darker corresponds to smaller $l_{0}$ (opposite to the ordering for $v_{2}, R_{\mathrm{AA}}$ and $\left.v_{2}^{I_{\mathrm{AA}}}\right)$.

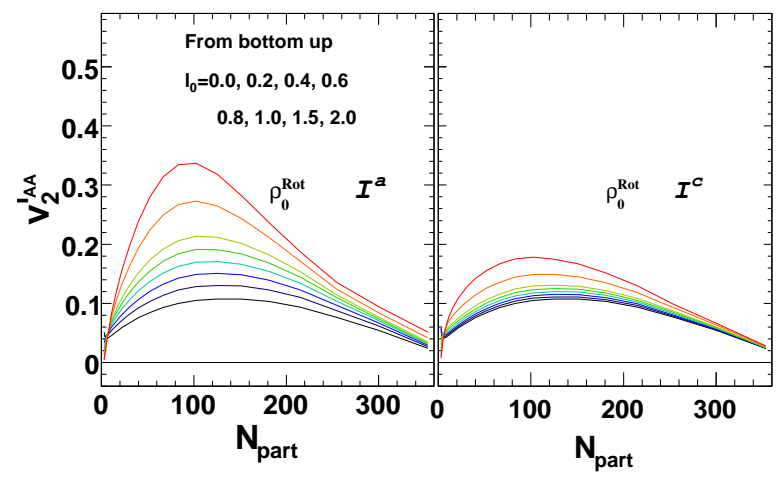

FIG. 23: Similar to Fig. 22] except plotted for $v_{2}^{I_{\mathrm{AA}}}$. Curves that are lower and darker corresponds to smaller $l_{0}$

jet quenching observables using a $\rho_{0}$ matter profile ${ }^{5}$ with $\kappa$ varied to allow the $R_{\mathrm{AA}}$ to change by $\pm 12 \%$ in the $0 \%$ $5 \%$ centrality bin for each $m$ value (panel (a)). These $\kappa$ values are then used to predict the centrality dependence

TABLE II: The $\kappa$ values for three matter profiles, $\rho_{0}$ (participant profile), $\rho_{2}$ (two component profile with $\delta=0.14$ ), and $\rho_{3}$ (CGC profile), and four $l$ dependencies $(m=1,2,3,4)$. The range of $\kappa$ in each case corresponds to $0.160<R_{\mathrm{AA}}<$ 0.204 in the $0 \%-5 \%$ centrality bin. The total matter integrals for each profile are also listed.

\begin{tabular}{l|l|l|l|l|l|}
\hline & Integral & $I_{1}$ & $I_{2}$ & $I_{3}$ & $I_{4}$ \\
\hline$\rho_{0}$ & 353 & $0.147_{-10 \%}^{+12 \%}$ & $0.082_{-14 \%}^{+18 \%}$ & $0.035_{-18 \%}^{+24 \%}$ & $0.0125_{-21 \%}^{+29 \%}$ \\
$\rho_{2}$ & 301 & $0.185_{-11 \%}^{+14 \%}$ & $0.117_{-15 \%}^{+20 \%}$ & $0.054_{-19 \%}^{+26 \%}$ & $0.0206_{-22 \%}^{+31 \%}$ \\
$\rho_{3}$ & 695 & $0.076_{-10 \%}^{+12 \%}$ & $0.046_{-14 \%}^{+19 \%}$ & $0.020_{-18 \%}^{+24 \%}$ & $0.0073_{-21 \%}^{+29 \%}$ \\
\hline
\end{tabular}

${ }^{5}$ Similar results are also observed for $\rho_{2}$ and $\rho_{3}$ matter profiles. 
of $v_{2}$ (panel (b)), $I_{\mathrm{AA}}\left(\right.$ panel (c)) and $v_{2}^{I_{\mathrm{AA}}}$ (panel (d)). We can see that the fractional change on $v_{2}$ is typically smaller than $5 \%$ for $N_{\text {part }}>100$, and the change decreases for larger $N_{\text {part }}$; the direction of the change, however, is anti-correlated with the change of $R_{\mathrm{AA}}$. The fractional change on $I_{\mathrm{AA}}$ is about $20 \%$, about twice amount of the change of $R_{\mathrm{AA}}$; this change is in the same direction as that for the $R_{\mathrm{AA}}$. The fractional change in $v_{2}^{I_{\mathrm{AA}}}$ is more complicated: It typically decreases for central collisions and increases for mid-central and peripheral collisions, but the overall change is typically less than $5 \%$.
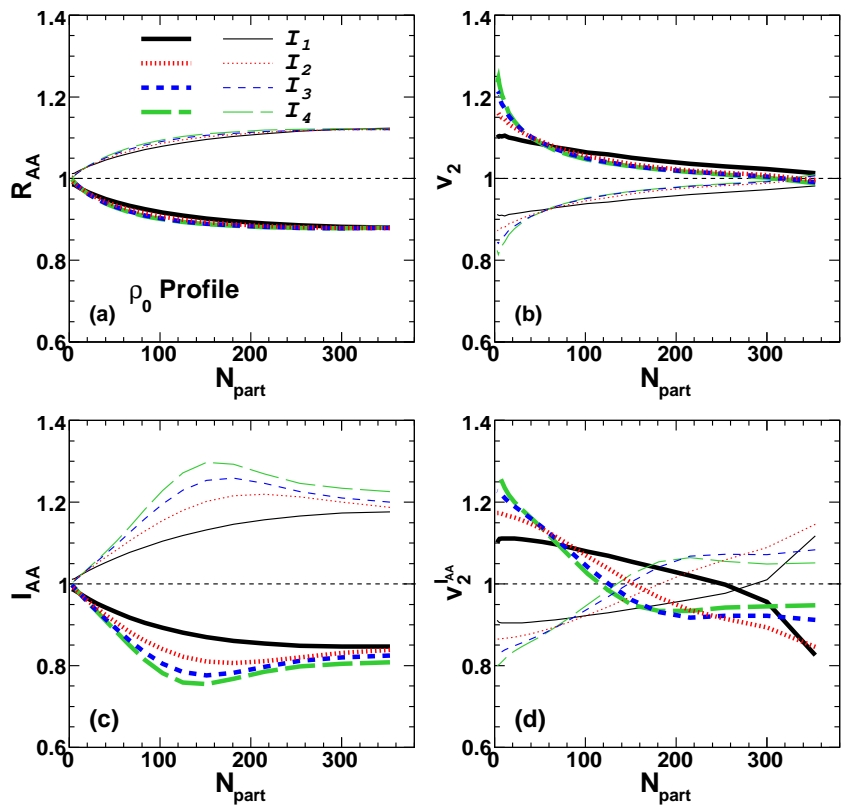

FIG. 24: (Color online) Centrality dependence of (a): $R_{\mathrm{AA}}$, (b) $v_{2}$, (c) $I_{\mathrm{AA}}$ and (d) $v_{2}^{I_{\mathrm{AA}}}$ using a $\rho_{0}$ matter profile with $\kappa$ varied to allow the $R_{\mathrm{AA}}$ to change by $\pm 12 \%$ in the $0 \%$ $5 \%$ centrality bin. Different line styles are used for $m=1$ (solid lines), $m=2$ (dotted lines), $m=3$ (dashed lines), and $m=4$ (long dashed lines); the upper (lower) values of $\kappa$ are represented by thin (thick) lines. Similar results are also observed for $\rho_{2}$ and $\rho_{3}$ matter profiles (not shown).

\section{Appendix C: Comments on finite nucleon size effect, event-by-event fluctuation etc}

We can show explicitly why the finite nucleon size is not important for $v_{2}$ calculation except in most peripheral collisions. We notice that the finite nucleon size leads to an increase of the variance matter profile, but does not change the orientation of the rotated frame:

$$
\begin{aligned}
\sigma_{x}^{\prime 2} & \rightarrow \sigma_{x}^{\prime 2}+r_{0}^{2} \\
\sigma_{y}^{\prime 2} & \rightarrow \sigma_{y}^{\prime 2}+r_{0}^{2} \\
\sigma_{x y}^{\prime}=0 & \rightarrow \sigma_{x y}^{\prime}=0 \\
\sigma_{r}^{\prime 2} & \rightarrow \sigma_{r}^{\prime 2}+r_{0}^{2}
\end{aligned}
$$

$$
\epsilon^{\mathrm{part}} \rightarrow \epsilon^{\mathrm{part}}\left(1+\frac{r_{0}^{2}}{\sigma_{r}^{2}}\right)
$$

The eccentricity decreases a little bit due to smearing of $n-n$ overlap function. However, for a typical fireball size of $\sigma_{r}=3 \mathrm{fm}$, this is only a $1.7 \%$ change in the eccentricity. ${ }^{6}$ The RMS size of the ellipsoid also increases by a few percent, but the corresponding jet production profile increases by the same amount, resulting in almost no change on the calculated $v_{2}$. Figure 25] shows the ratio of the $v_{2}$ for event averaged $\rho_{0}$ calculated assuming the nucleon profile follows either the $\delta$ function or a Gauss function with width of $r_{0}=0.4 \mathrm{fm}$. The differences of $v_{2}$ are well within $3 \%$, except at $N_{\text {part }}<20$, where the $r_{0}=0.4 \mathrm{fm}$ case is smaller.
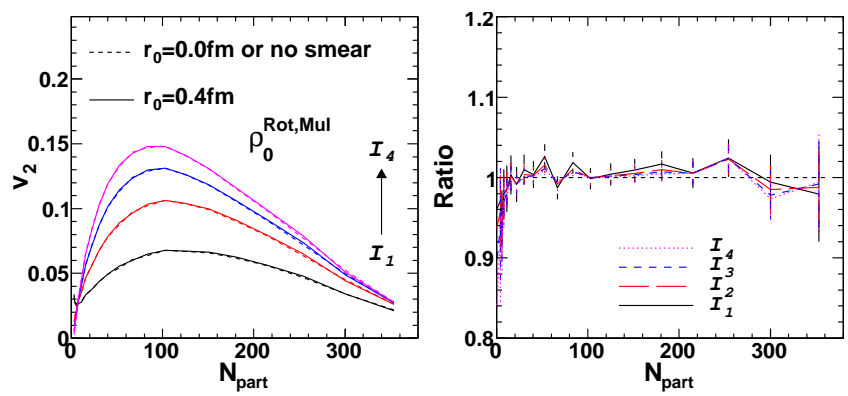

FIG. 25: (Color online) Comparison of the $v_{2}$ 's for Glauber geometry, which are filled either according to center positions of nucleons or assuming Gauss profiles with a width of $0.4 \mathrm{fm}$.

Note that similar findings also appear in MC-KLN, which assumes that participating nucleons are disks of finite size in filling the participant profile distribution (in addition to assuming finite nucleon size for determining whether it is a participant). This is illustrated by Fig. 26, which compares the $v_{2}$ results for the same Glauber geometry determined with either the PHOBOS code or the MC-KLN code. The ratio drops at $N_{\text {part }}<20$ similar to Fig. 25. As a side note, we point out that this plot also confirmed the consistency between the PHOBOS code and the MC-KLN code for calculating the Glauber geometry when running with the same parameters.

We have seen that the event-by-event fluctuation leads to large dispersion of the distribution of various geometrical variables, such as eccentricity and RMS size in Fig.13. Yet the mean values seem to be insensitive to whether they are calculated for each event and then averaged over many events or are calculated directly from the averaged profile. As an example, Fig. 27 compares the eccentricity averaged over values calculated event by event $\left\langle\frac{\sigma_{y}^{\prime 2}-\sigma_{x}^{\prime 2}}{\sigma_{y}^{\prime 2}+\sigma_{x}^{\prime 2}}\right\rangle$ with the eccentricity calculated from the average profile $\frac{\left\langle\sigma_{y}^{\prime 2}\right\rangle-\left\langle\sigma_{x}^{\prime 2}\right\rangle}{\left\langle\sigma_{y}^{\prime \prime}\right\rangle+\left\langle\sigma_{x}^{\prime 2}\right\rangle}$. The ratios are shown in the right panel for

\footnotetext{
${ }^{6}$ The impact is somewhat larger for $\epsilon_{\mathrm{RP}}$, i.e. average without rotation
} 
both $\epsilon_{\mathrm{RP}}$ and $\epsilon_{\mathrm{part}}$. As one can see, the difference is $<2 \%$ for $\epsilon_{\mathrm{part}}$ and somewhat larger for $\epsilon_{\mathrm{RP}}{ }^{7}$. This is quite remarkable given the sharp visual contrast between the lumpiness of the event-by-event profile and the smoothness of the average profile (see Fig. 28).
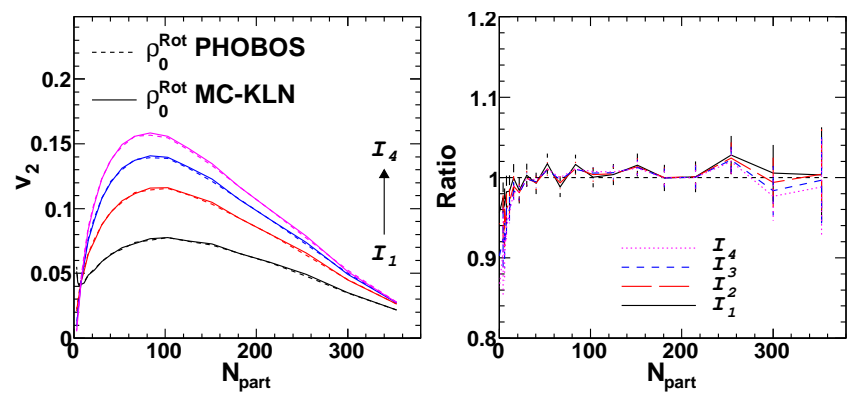

FIG. 26: (Color online) Comparison of the $v_{2}$ 's for Glauber geometry calculated using the PHOBOS code and the MCKLN code.
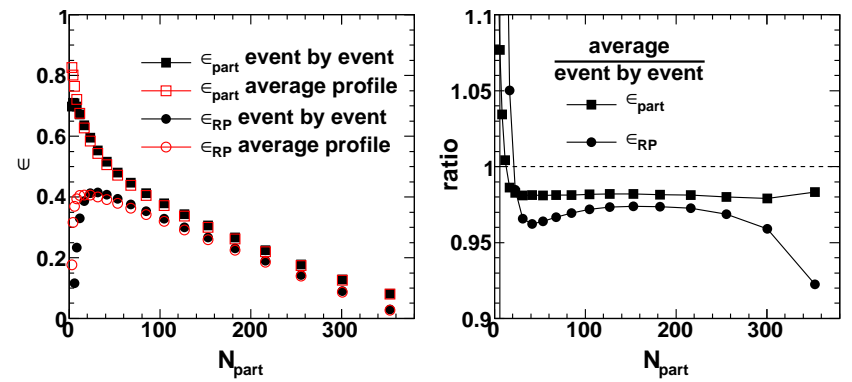

FIG. 27: (Color online) Left panel: the standard (circles) and participant (boxes) eccentricity either by averaging over their event-by-event value (filled symbols) or calculated directly from the averaged matter profiles (open symbols). Right panel: the corresponding ratios for the standard and participant eccentricities.

7 The agreement generally worsens when the width of the distribution becomes large relative to the mean value, which is the
Nevertheless, the good news is that the average profile seems to preserve most of the relevant geometrical information. For example, one can calculate the eccentricity directly from the overall matter profile instead of calculating it event by event and then averaging it over many events.

case for $\epsilon_{\mathrm{RP}}$.

[1] K. Adcox et al., Nucl. Phys. A 757 (2005) 184; J. Adams et al., Nucl. Phys. A 757 (2005) 102; B. B. Back et al., Nucl. Phys. A 757 (2005) 28; I. Arsene et al., Nucl. Phys. A 757 (2005) 1.

[2] M. Gyulassy, I. Vitev, X. N. Wang and B. W. Zhang, arXiv:nucl-th/0302077

[3] U. A. Wiedemann, arXiv:0908.2306 [hep-ph].

[4] A. Majumder and M. Van Leeuwen, arXiv:1002.2206 [hep-ph].

[5] K. Adcox et al., Phys. Rev. Lett. 88, 022301 (2002)

[6] I. Vitev and M. Gyulassy, Phys. Rev. Lett. 89, 252301 (2002)

[7] A. Adare et al., Phys. Rev. C 78, 014901 (2008)

[8] H. Zhang, J. F. Owens, E. Wang and X. N. Wang, Phys. Rev. Lett. 98, 212301 (2007)

[9] A. Adare et al., Phys. Rev. C 80, 024908 (2009)
[10] B. I. Abelev et al. arXiv:0912.1871 [nucl-ex].

[11] H. Zhang, J. F. Owens, E. Wang and X. N. Wang, Phys. Rev. Lett. 103, 032302 (2009)

[12] G. Y. Qin, J. Ruppert, C. Gale, S. Jeon and G. D. Moore, Phys. Rev. C 80, 054909 (2009)

[13] S. A. Bass et al. Phys. Rev. C 79, 024901 (2009)

[14] B. Muller, Prog. Theor. Phys. Suppl. 174, 103 (2008).

[15] S. Peigne and A. V. Smilga, Phys. Usp. 52, 659 (2009) [Usp. Fiz. Nauk 179, 697 (2009)]

[16] E. V. Shuryak, Phys. Rev. C 66, 027902 (2002)

[17] A. Drees, H. Feng and J. Jia, Phys. Rev. C 71, 034909 (2005)

[18] R. Wei, Nucl. Phys. A 830, 175C (2009); A. Adare et.al., arXiv:1006.3740 [nucl-ex].

[19] A. Adare et al. Phys. Rev. Lett. 98, 172301 (2007)

[20] W. A. Horowitz and M. Gyulassy, Phys. Lett. B 666, 320 

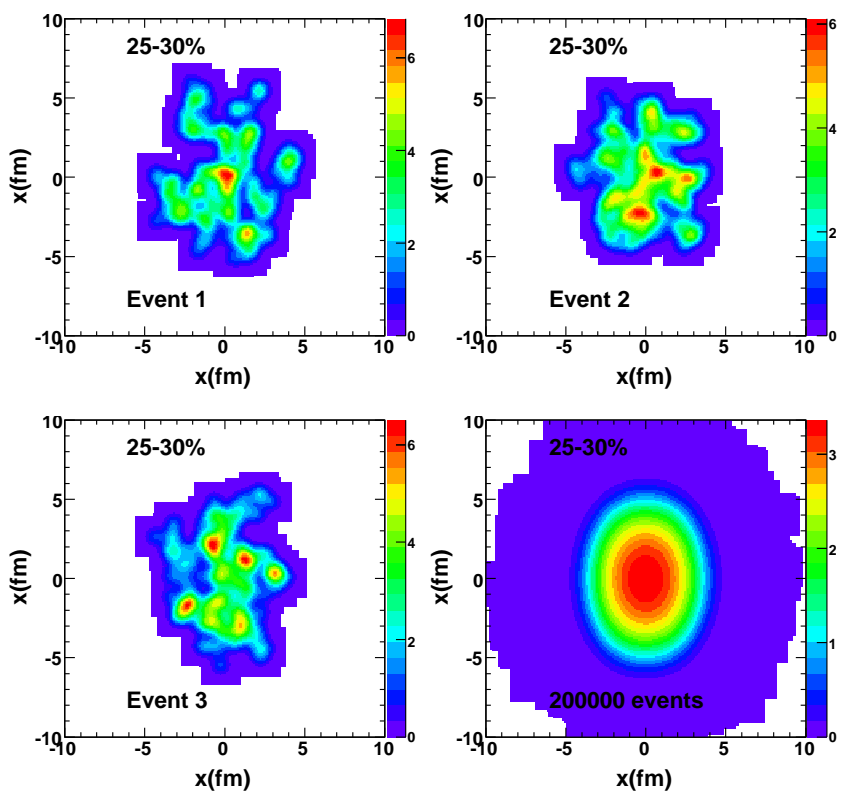

FIG. 28: (Color online) The event-by-event participant profiles $\left(\rho_{0}^{\text {Rot }}\right)$ for three typical events and profile averaged over 200,000 events for 25\%-30\% centrality selection. Nucleons are assumed to have a Gaussian profile with a width of $r_{0}=0.4$ $\mathrm{fm}$. All events have been shifted to center of gravity and rotated to participant plane.

(2008)

[21] S. S. Gubser, D. R. Gulotta, S. S. Pufu and F. D. Rocha, JHEP 0810, 052 (2008); P. M. Chesler, K. Jensen, A. Karch and L. G. Yaffe, Phys. Rev. D 79, 125015 (2009)

[22] F. Dominguez, C. Marquet, A. H. Mueller, B. Wu and B. W. Xiao, Nucl. Phys. A 811, 197 (2008)

[23] H. Liu, K. Rajagopal and U. A. Wiedemann, Phys. Rev. Lett. 97, 182301 (2006)

[24] C. Marquet and T. Renk, Phys. Lett. B 685, 270 (2010)

[25] J. Liao and E. Shuryak, Phys. Rev. Lett. 102, 202302 (2009)

[26] M. L. Miller, K. Reygers, S. J. Sanders and P. Steinberg, Ann. Rev. Nucl. Part. Sci. 57, 205 (2007)

[27] H. J. Drescher, A. Dumitru, A. Hayashigaki and Y. Nara, Phys. Rev. C 74, 044905 (2006)

[28] B. Alver et al., Phys. Rev. C 77, 014906 (2008)

[29] H. J. Drescher and Y. Nara, Phys. Rev. C 75, 034905 (2007)

[30] T. Hirano and Y. Nara, Phys. Rev. C 79, 064904 (2009)

[31] W. Broniowski, P. Bozek and M. Rybczynski, Phys. Rev. C 76, 054905 (2007)

[32] S. Afanasiev et al., Phys. Rev. C 80, 054907 (2009)

[33] R. A. Lacey et al., Phys. Rev. Lett. 103, 142302 (2009); Phys. Rev. C 80, 051901 (2009)

[34] B. Alver, M. Baker, C. Loizides and P. Steinberg, arXiv:0805.4411 [nucl-ex].
[35] D. Kharzeev, E. Levin and M. Nardi, Nucl. Phys. A 730, 448 (2004) [Erratum-ibid. A 743, 329 (2004)]

[36] U. W. Heinz, J. S. Moreland and H. Song, Phys. Rev. C 80, 061901 (2009)

[37] D. Kharzeev and M. Nardi, Phys. Lett. B 507, 121 (2001)

[38] B. B. Back et al. Phys. Rev. C 65, 061901 (2002)

[39] P. F. Kolb and U. W. Heinz, arXiv:nucl-th/0305084.

[40] A. K. Chaudhuri, arXiv:0801.3180 [nucl-th].

[41] A. Adare et al., Phys. Rev. Lett. 101, 232301 (2008)

[42] D. A. Teaney, arXiv:0905.2433 [nucl-th].

[43] S. S. Adler et al. Phys. Rev. C 71, 034908 (2005) [Erratum-ibid. C 71, 049901 (2005)]

[44] B. Alver et al. Phys. Rev. C 80, 011901 (2009)

[45] I. Vitev, Phys. Lett. B 639, 38 (2006)

[46] S. Wicks, W. Horowitz, M. Djordjevic and M. Gyulassy, Nucl. Phys. A 784, 426 (2007)

[47] A. Dainese, C. Loizides and G. Paic, Eur. Phys. J. C 38, 461 (2005)

[48] T. Renk, J. Ruppert, C. Nonaka and S. A. Bass, Phys. Rev. C 75, 031902 (2007)

[49] C. Nonaka and S. A. Bass, Phys. Rev. C 75, 014902 (2007)

[50] J. L. Nagle, P. Steinberg and W. A. Zajc, Phys. Rev. C 81, 024901 (2010)

[51] A. B. Migdal, Phys. Rev. 103, 1811 (1956).

[52] P. F. Kolb, J. Sollfrank and U. W. Heinz, Phys. Rev. C 62, 054909 (2000)

[53] K. J. Eskola, H. Honkanen, C. A. Salgado and U. A. Wiedemann, Nucl. Phys. A 747, 511 (2005)

[54] M. Luzum and P. Romatschke, Phys. Rev. C 78, 034915 (2008) [Erratum-ibid. C 79, 039903 (2009)]

[55] J. Vredevoogd and S. Pratt, Phys. Rev. C 79, 044915 (2009)

[56] B. Schenke, C. Gale and S. Jeon, Phys. Rev. C 80, 054913 (2009)

[57] A. Majumder, C. Nonaka and S. A. Bass, Phys. Rev. C 76, 041902 (2007)

[58] N. Armesto, M. Cacciari, T. Hirano, J. L. Nagle and C. A. Salgado, J. Phys. G 37, 025104 (2010)

[59] V. S. Pantuev, JETP Lett. 85, 104 (2007)

[60] I. P. Lokhtin, A. M. Snigirev and I. Vitev, arXiv:hep-ph/0212061

[61] T. Renk and K. Eskola, Phys. Rev. C 75, 054910 (2007)

[62] L. Frankfurt, M. Strikman and C. Weiss, Phys. Rev. D 69, $114010(2004)$

[63] X. D. Ji, Phys. Rev. Lett. 91, 062001 (2003)

[64] We have placed the two-dimensional profile functions in $5 \%$ centrality bin for participant density, collision density and gluon density with eccentricity fluctuation at https://www.phenix.bnl.gov/WWW/publish/jjia /glauber/profile/profile.html. They can be used as input for viscous hydrodynamics and realistic jet quenching model calculations.

[65] A. Adare et al., Phys. Rev. Lett. 104, 252301 (2010)

[66] J. Adams et al., Phys. Rev. Lett. 97, 162301 (2006)

[67] J. Jia, Int. J. Mod. Phys. E 16, 2000 (2007) 